This is a self-archived version of an original article. This version may differ from the original in pagination and typographic details.

Author(s): Ouchakour, Lamiaa; Ábrahámi, Renáta A.; Forró, Enikő; Haukka, Matti; Fülöp,
Ferenc; Kiss, Lorand

Title: Stereocontrolled synthesis of fluorine-containing piperidine $y$-amino acid derivatives

Year: 2019

Version: Accepted version (Final draft)

Copyright: @ 2019 WILEY

Rights: In Copyright

Rights url: http://rightsstatements.org/page/InC/1.0/?language=en

Please cite the original version:

Ouchakour, L., Ábrahámi, R. A., Forró, E., Haukka, M., Fülöp, F., \& Kiss, L. (2019).

Stereocontrolled synthesis of fluorine-containing piperidine $\gamma$-amino acid derivatives. European Journal of Organic Chemistry, 2019(12), 2202-2211. https://doi.org/10.1002/ejoc.201801540 


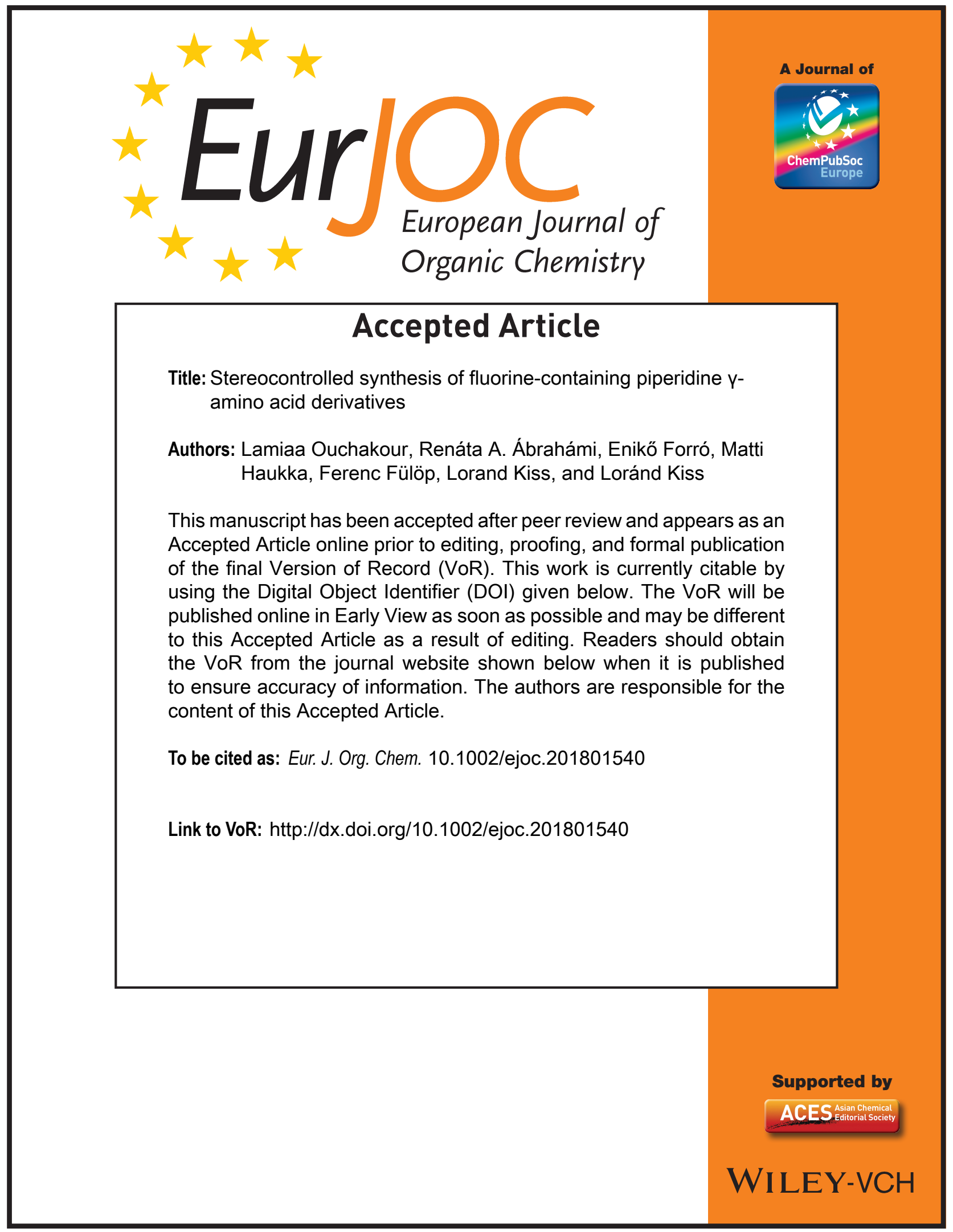




\title{
Stereocontrolled synthesis of fluorine-containing piperidine $\gamma$-amino acid derivatives
}

\author{
Lamiaa Ouchakour, ${ }^{[a],[c]}$ Renáta A. Ábrahámi, ${ }^{[a]}$ Enikő Forró, ${ }^{[a]}$ Matti Haukka, ${ }^{[d]}$ Ferenc \\ Fülöp, ${ }^{[\mathrm{a}],[\mathrm{b}],[\mathrm{c}]}$ Loránd Kiss $^{[\mathrm{a}],[\mathrm{c}] *}$
}

${ }^{[a]}$ Institute of Pharmaceutical Chemistry, University of Szeged, H-6720 Szeged, Eötvös u. 6 , Hungary

${ }^{[b]}$ MTA-SZTE Stereochemistry Research Group, Hungarian Academy of Sciences, H-6720 Szeged, Eötvös u. 6, Hungary

${ }^{[c]}$ University of Szeged, Interdisciplinary Excellence Centre, Institute of Pharmaceutical Chemistry

${ }^{[d]}$ Department of Chemistry, University of Jyväskylä, FIN-40014, Jyväskylä, Finland

E-mail: kiss.lorand@ pharm.u-szeged.hu; kiss.lorand00@gmail.com

http://www2.pharm.u-szeged.hu/gyki

\section{Graphical abstract:}

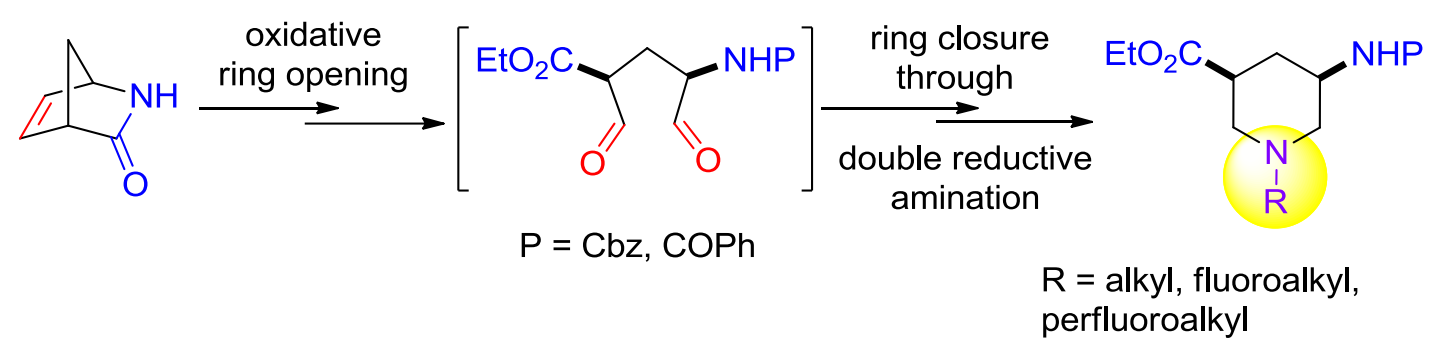

\begin{abstract}
An efficient synthetic approach for the construction of fluorine-containing piperidine $\gamma$-amino acid derivatives has been developed. The synthetic concept was based on oxidative ring opening of an unsaturated bicyclic $\gamma$-lactam (Vince-lactam) through its ring $\mathrm{C}=\mathrm{C}$ bond, followed by double reductive amination of the diformyl intermediate performed with various fluoroalkylamines. The method has been extended towards the access of alkylated and perfluoroalkylated substances and for $\gamma$-lactam derivatives. The transformations proceeded with stereocontrol: the configuration of the stereocenters in the products were predetermined by the configuration of the chiral centers of the starting $\gamma$-lactam. The method could be extended for the access to enantiopure piperidine $\gamma$-amino esters.
\end{abstract}


Keywords: amino acids, piperidines, ring closing, reductive amination, fluorine

\section{Introduction}

Although less abundant than their $\alpha$-analogs, $\gamma$-amino acids are considered to be an important family of compounds in medicinal chemistry. Representatives of this class of compounds include some acyclic derivatives, such as the neurotransmitter GABA $(\gamma-$ aminobutyric acid), $(R)$-Baclofen the agonist of the $\mathrm{GABA}_{\mathrm{C}}$ receptor, $(S)$-Vigabatrin an inhibitor of GABA-T or $(S)$-Pregabalin an antiepileptic, antihyperalgesic agent (Figure 1). ${ }^{[1]}$<smiles>NCCCC(=O)O</smiles>

GABA (1)<smiles>NCC(CC(=O)O)c1ccc(Cl)cc1</smiles>

$(R)$-Baclofen (2)<smiles>C=CC(N)CCC(=O)O</smiles>

(S)-Vigabatrin (3)<smiles>CC(C)CC(CN)CC(=O)O</smiles>

(S)-Pregabalin (4)

Figure 1. Some bioactive acyclic $\gamma$-amino acids.

Alicyclic $\gamma$-amino acids are conformationally rigid analogs of their acylic counterparts, some of them are known as $\mathrm{GABA}_{\mathrm{A}}$ or $\mathrm{GABA}_{\mathrm{C}}$ receptors, while Gabapentin is a drug commercialized for the treatment of cerebral disorder diseases (Figure 2). ${ }^{[1]}$ Furthermore, cyclic $\gamma$-amino acids as conformationally restricted structures are key building elements in the synthesis of pharmaceutically relevant $\alpha / \gamma-, \beta / \gamma$ - or $\gamma / \gamma$-peptides. ${ }^{[2]}$<smiles>NC1C=CC(C(=O)O)C1</smiles>

5

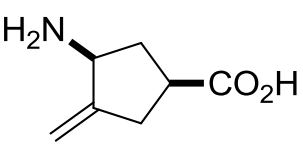

6<smiles>NC1CC=C(C(=O)O)C1</smiles>

7<smiles>NCC1(CC(=O)O)CCCCC1</smiles>

Gabapentin (8)

Figure 2. Structures of several biologically relevant alicyclic $\gamma$-amino acids.

As a result of the considerable importance of fluorinated organic molecules in medicinal chemistry and the increasing impact of fluorine-containing biomolecules in drug research, an ever-increasing number of fluorination methodologies have emerged during the past decade for the access of versatile fluorine-containing molecular entities. Among them, fluorinated amino acids are of significant importance in drug research since they might exhibit more efficient 
biological activities than their non-fluorinated counterparts. ${ }^{[3]}$ Some fluorine-containing cyclic $\gamma$-amino acid representatives (Figure 3) possess relevant biological properties; e.g. they are potential inactivators of $\gamma$-aminobutyric acid aminotransferase (GABA-AT) (Figure 3). ${ }^{[4]}$
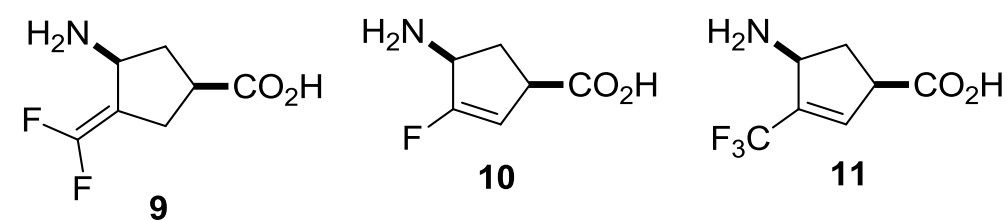

Figure 3. Biologically interesting alicyclic fluorine-containing $\gamma$-amino acids.

The antiviral drug Peramivir ${ }^{[5]}$ contains a functionalized cyclopentane $\gamma$-amino acid motif whose synthesis starts from bicyclic $\gamma$-lactam 2-azabicyclo[2.2.1]hept-5-ene-3-one (Vince lactam, $( \pm)-15) .{ }^{[6]}$ Vince lactam, a valuable building block, is the starting material for the access of bioactive carbasugars and amino acid carbasugars. Furthermore, it is the precursor in the synthesis of some blockbuster drugs such as of carbovir, abacavir or entecavir. ${ }^{[7]}$

Some heterocyclic $\alpha$-amino acid derivatives with a piperidine moiety possess various biological properties. For example, Remifentanil and Carfentanil with a piperidine 4-amino-4-carboxylate framework are anesthetics, while AZD5363 is an antitumoral agent (Figure 4). ${ }^{[1 \mathrm{~h}, \mathrm{i}],[8]}$ Apart from this, some five- and six-membered azacyclic $\beta$-amino acids, possessing both the ring $N$ atom and an extracyclic amino group in their structures, as small molecular entities exhibit antiviral activities or they are key components of bioactive substances with complex structures with antitumoral or antibacterial properties. ${ }^{[9]}$

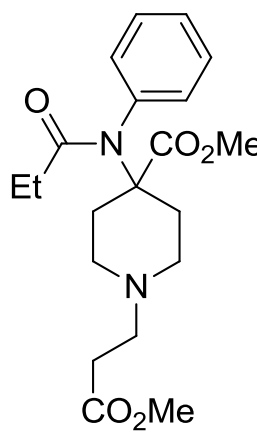

Remifentanil (12)

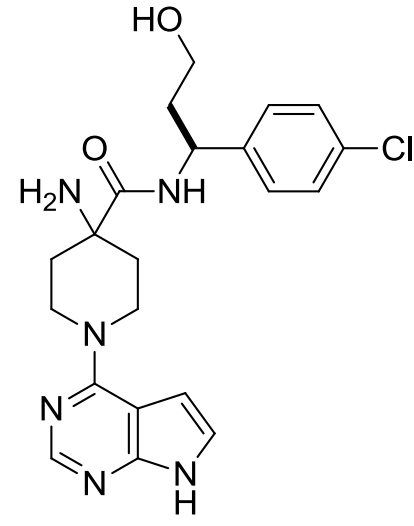

AZD5363 (13)<smiles>CCC(=O)N(c1ccccc1)C1(C(=O)OC)CCN(CCc2ccccc2)CC1</smiles>

Carfentanil (14)

Figure 4. Some bioactive $\alpha$-amino acid derivatives with a piperidine core. 
In view of the high biological potential of both organofluorine derivatives and $\gamma$-amino acids, we have planned to develop a synthetic protocol towards the access of novel fluorinated piperidine $\gamma$-amino acid derivatives.

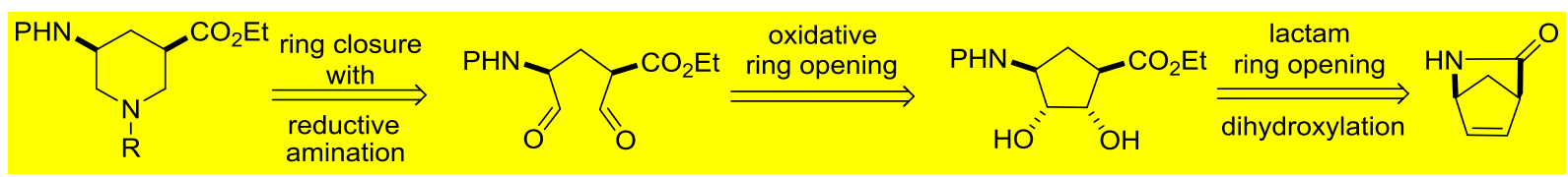

Scheme 1.

Our synthetic strategy was based on the application of an oxidative ring-opening/reductive ringclosure protocol. ${ }^{[9 c, 10]}$ Specifically, we carried out oxidative ring cleavage via the $\mathrm{C}-\mathrm{C}$ double bond of bicyclic Vince lactam (Scheme 1) followed by double reductive amination through ring closing with various fluorine-containing amines.

\section{Results and Discussion}

Racemic Vince lactam ( \pm )-15 was first subjected to acid-catalyzed heteroring opening with ethanolysis followed by $\mathrm{Cbz}$ or benzoyl protection of the amino group to have the corresponding cyclopentene $\gamma$-amino esters $( \pm)-16$ and $( \pm)-17$.

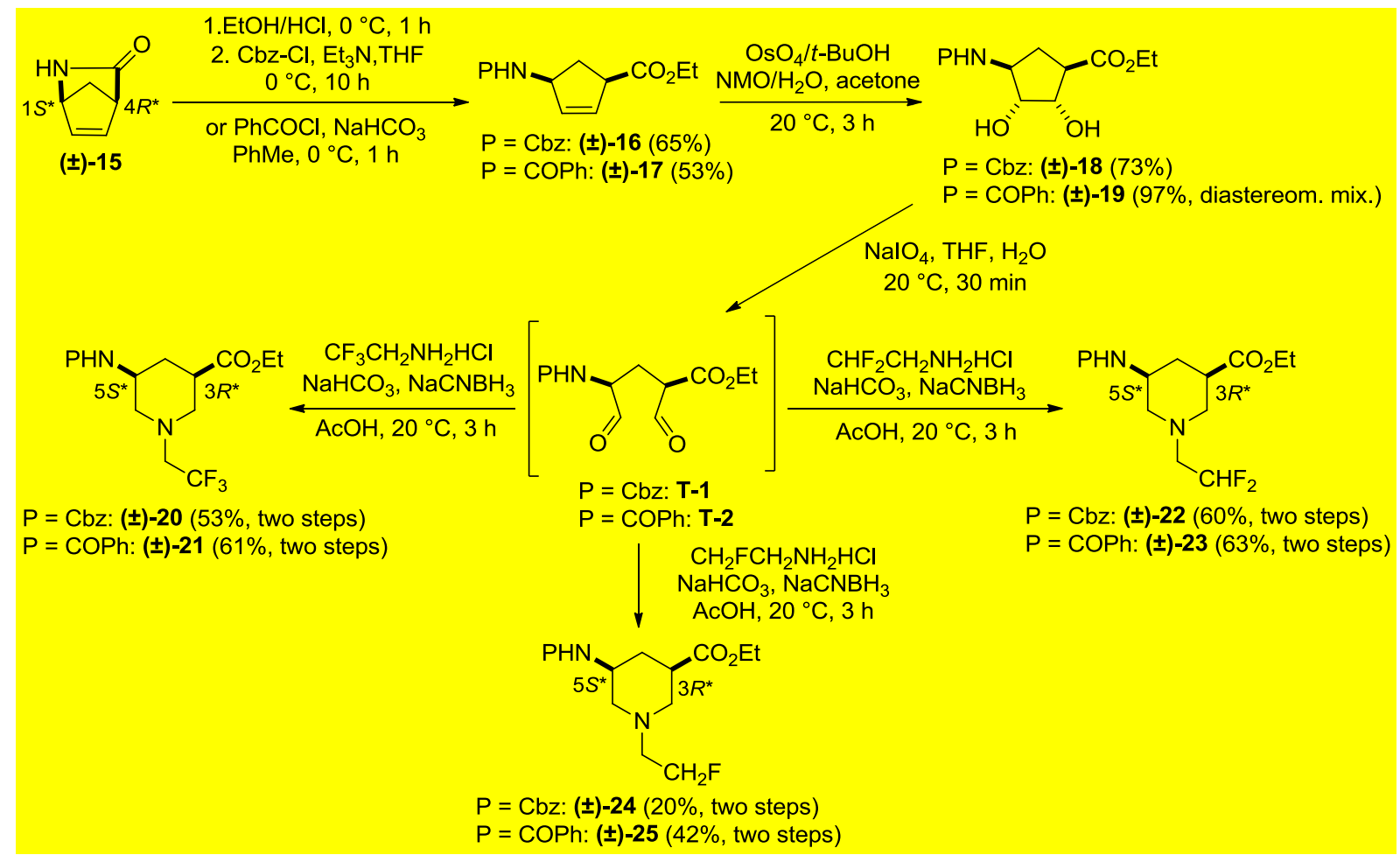

Scheme 2. 
Next, these compounds were oxidized with $\mathrm{NMO}$ and a catalytic amount of $\mathrm{OsO}_{4}$. While cis-dihydroxylation of Cbz-protected ester $( \pm)-16$ diastereoselectively produced diol $( \pm)-18$ (with the hydroxy groups, the ester and carbamate functions in trans relationship), benzoylprotected derivative $( \pm)-\mathbf{1 7}$ furnished a mixture of diols [cis/trans $( \pm)-19]$ in nearly 1:1 ratio.

In continuation, both $( \pm)$-18 and diastereomeric mixture $( \pm)$-19 were submitted to a onepot transformation, which included oxidative ring cleavage with $\mathrm{NaIO}_{4}$ followed by double reductive amination with fluorine-containing amines. Oxidative ring opening of diols ( \pm )-18 and $( \pm)-19$ led to the corresponding unstable dialdehyde derivatives T-1 and T-2 which, in turn, were immediately transformed further after work-up of the oxidative ring-cleavage step. The reductive amination steps were performed by the addition of the fluorine-containing amine $\mathrm{HCl}$ salts (mono-, di- or trifluoroethylamines) in the presence of $\mathrm{NaHCO}_{3}$ in a $\mathrm{CH}_{2} \mathrm{Cl}_{2} / \mathrm{THF}$ solvent mixture followed by the addition of $\mathrm{NaCNBH}_{3}$ at room temperature, after $10 \mathrm{~min}$ in the presence of $\mathrm{AcOH}$ (see Experimental). This order of addition of the reagents proved to be essential in these reactions. In all cases, reductive aminations took place with ring expansion providing the corresponding racemic trifluorinated $[( \pm)-20$ and $( \pm)-21]$, difluorinated $[( \pm)-22$ and ( \pm )-23], and monofluorinated piperidine $\gamma$-amino esters $[( \pm)-24$ and $( \pm)-25]$ (Scheme 2, Figure 5).

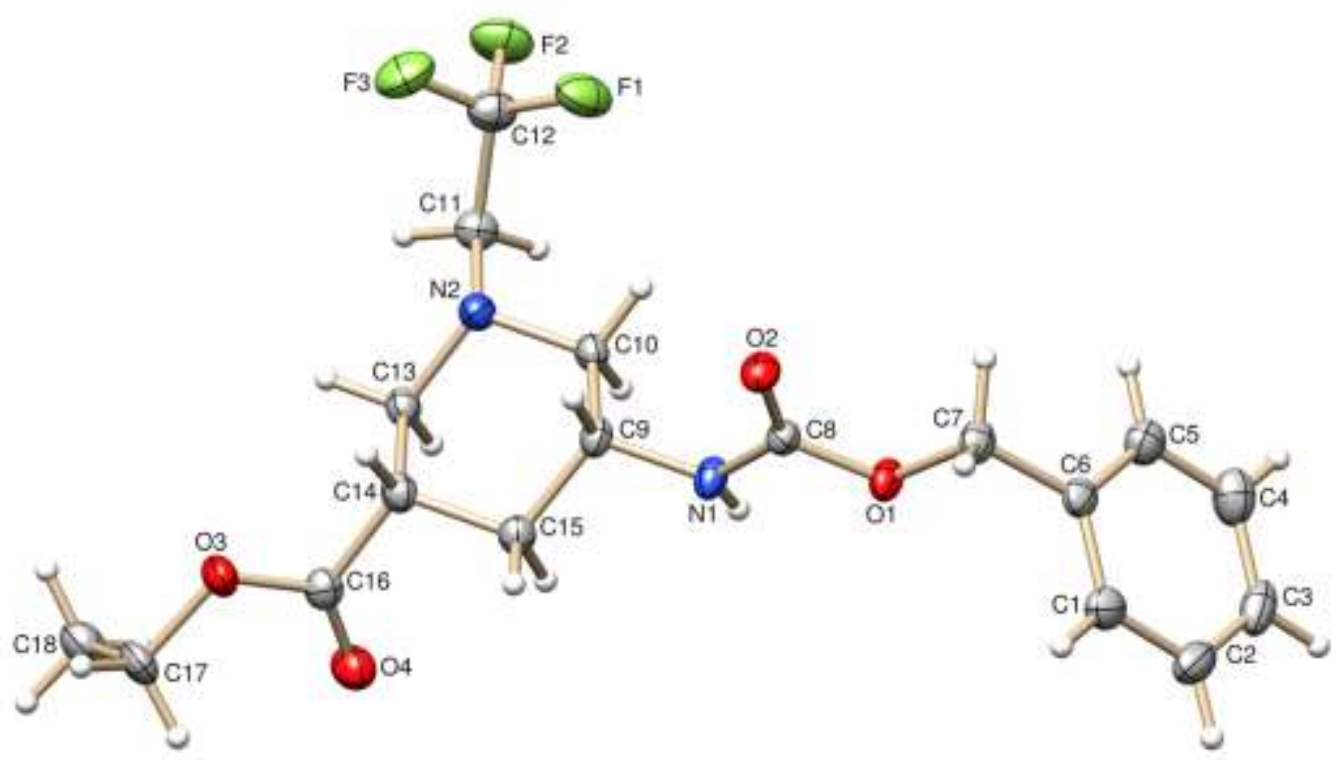

Figure 5. X-ray structure of compound $( \pm)-20$.

Noteworthy, the synthetic protocol proceeded with stereocontrol, that is the configurations of the chiral centers in all products were predetermined by the configuration of the stereocenters of starting lactam $( \pm)-\mathbf{1 5}$. 
Note, that the dialdehyde intermediates (T-1 and T-2) possess active hydrogens in the $\alpha$ position to the formyl moieties. Fortunately, the possible enolization, which would lead through the cyclization process to inversion of configuration with the piperidine ring containing the ester and protected amine in trans relationship, did not take place. The preferred diequatorial arrangement of both the ester and amino groups might be responsible for the conservation of the relative stereochemistry that is the formation of products bearing the two groups in cis relative arrangement (see structures T-5 and T-6, Scheme 3).

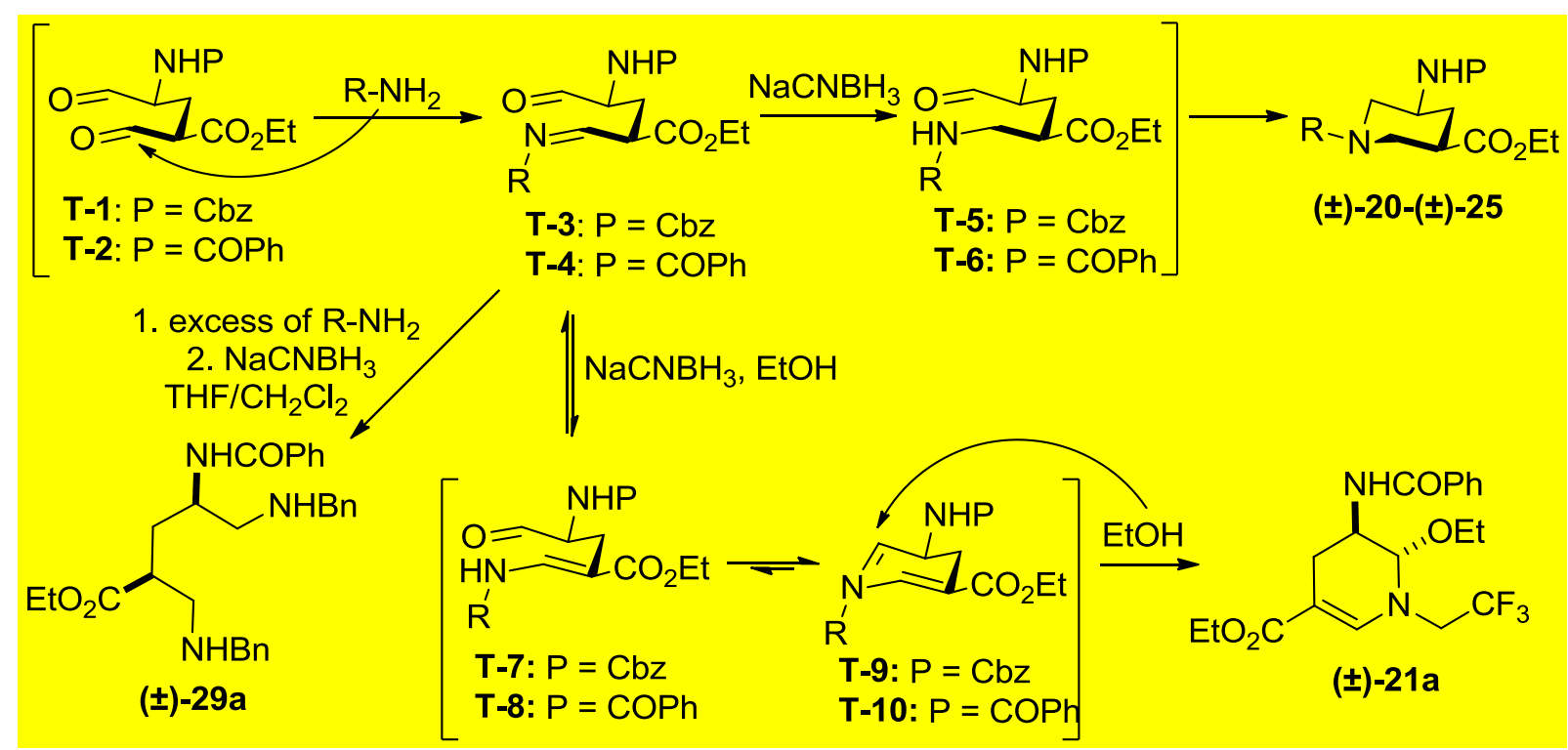

Scheme 3.

Next we planned to extend the synthetic protocol based on oxidative cleavage/ring closure for the access of other novel functionalized piperidines. Thus, nonafluoropentanamine, trifluoropropylamine and benzylamine were used as amine sources in the ring-closing step with dialdehydes T-1 or T-2. The addition of $\mathrm{NaCNBH}_{3}$ in $\mathrm{THF} / \mathrm{CH}_{2} \mathrm{Cl}_{2}$ after 10 min provided the corresponding $\gamma$-amino esters $( \pm)-26,( \pm)$-27, $( \pm)$-28 and $( \pm)$-29 through double reductive amination (Table 1). Note that the ring closure of T-2 with trifluoroethylamine was carried out in EtOH as solvent, adduct $( \pm)$-21a was formed through the nucleophilic attack of EtOH (Table 1), rather than piperidine derivative $( \pm)$-21 observed earlier. As mentioned, the order and timing of addition of the reagents were essential. When $\mathrm{NaCNBH}_{3}$ was added immediately to the mixture of dialdehyde and amine, the ring closure did not occur; instead, the corresponding diamino derivative ( \pm )-29a was obtained (Table 1$)$. 


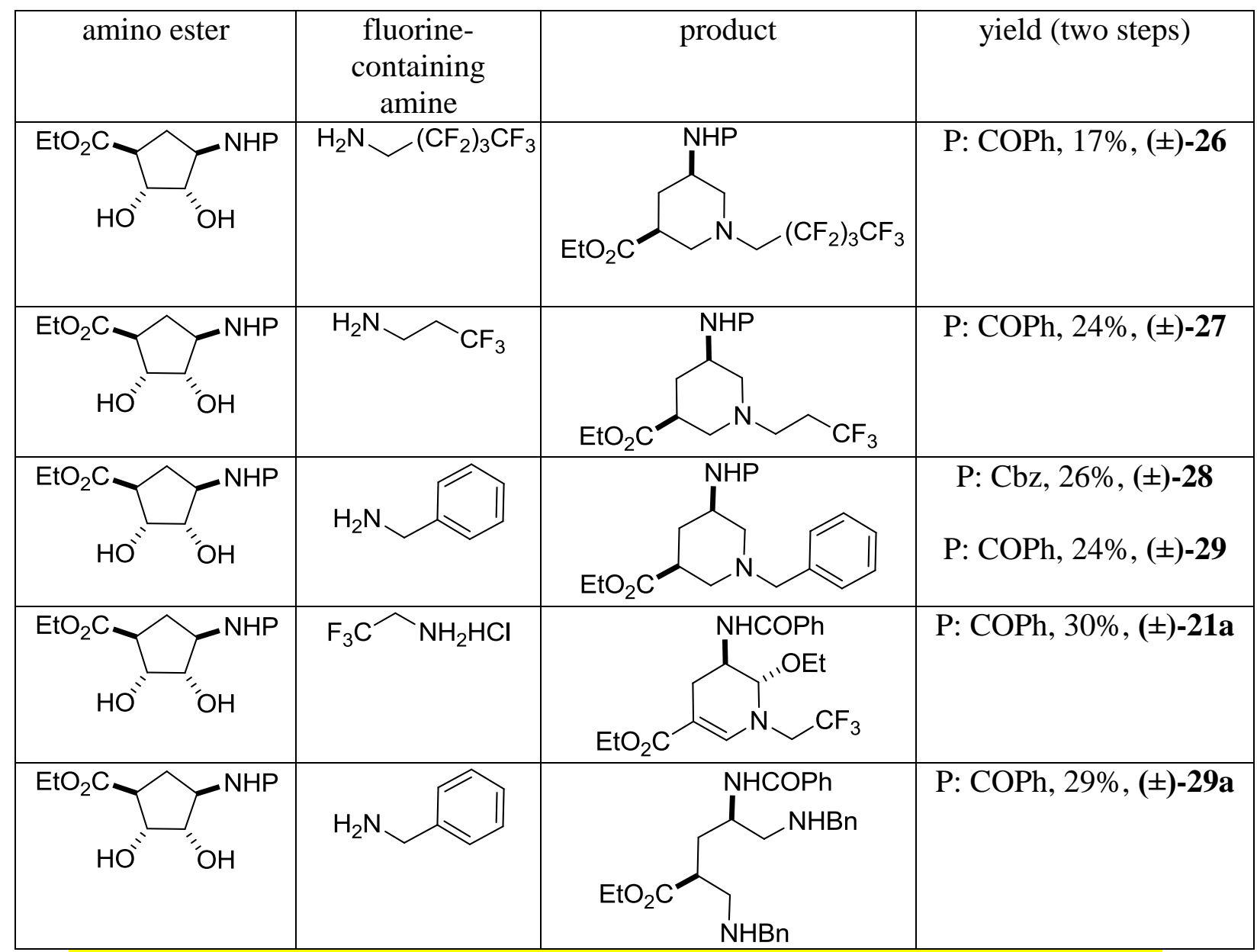

Table 1. Synthesis of piperidine derivatives $( \pm)-21 a,( \pm)-26-( \pm)-28,( \pm)-29 a$. Reaction conditions: $\mathrm{NaCNBH}_{3}, \mathrm{CH}_{2} \mathrm{Cl}_{2}, 20^{\circ} \mathrm{C}$ (see experimental part).

In continuation, we designed the synthesis of novel piperidine $\gamma$-amino ester derivatives. In order to increase the number of six-membered $N$-heterocyclic $\gamma$-amino ester isomers, first the isomerization of the ring double bond in $( \pm)-\mathbf{1 6} /( \pm)$-17 was accomplished. Compounds $( \pm)-$ 16/( \pm -17 underwent ring double bond migration through the deprotonation of the active $\mathrm{H}$ in the presence of NaOEt leading via isomerization to unsaturated esters $( \pm)-\mathbf{3 0}$ and $( \pm)-31$. Diol derivatives ( \pm )-32 and $( \pm)$-33, obtained by $\mathrm{OsO}_{4}$-catalyzed cis-stereoselective dihydroxylation of $( \pm)$-30 and $( \pm)-31$ (determined by the NMR analysis of the crude mixture), were next subjected to oxidative ring opening with $\mathrm{NaIO}_{4}$ in $\mathrm{THF} / \mathrm{H}_{2} \mathrm{O}$ (Scheme 4).

The resulting dicarbonyl intermediates T-11/T-12 were immediately submitted to the reaction with trifluoroethylamine without isolation. In the first step, most probably, the attack of the amine takes place at the $\alpha$-oxo group (which leads to an enamine with a conjugated system). 


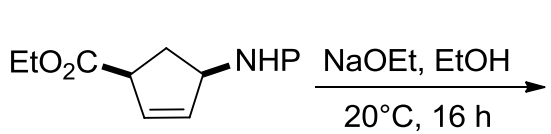

(士)-16: $\mathrm{P}=\mathrm{Cbz}$

(士)-17: $P=C O P h$

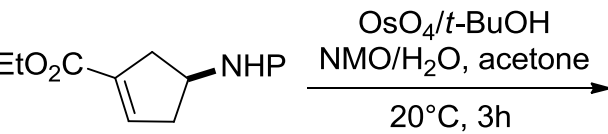

(士)-30: $\mathrm{P}=\mathrm{Cbz}(30 \%)$

$( \pm)-31: P=\operatorname{COPh}(33 \%)$

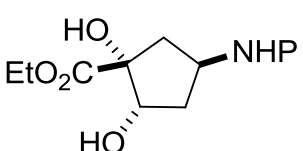

(士)-32: $\mathrm{P}=\mathrm{Cbz}(65 \%)$

(士)-33: $P=$ COPh $(59 \%)$ $\mathrm{NalO}_{4}, \mathrm{THF} / \mathrm{H}_{2} \mathrm{O}$ $20^{\circ} \mathrm{C}, 30 \mathrm{~min}$<smiles>CCOC(=O)NC1CCN(CC(F)(F)F)C(CC(C)(F)F)C1</smiles>

(士)-34: (25\%, two steps)<smiles>CCOC(=O)C1=CC(NC(=O)c2ccccc2)CCN1CC(F)(F)F</smiles>

(士)-35: (23\%, two steps)

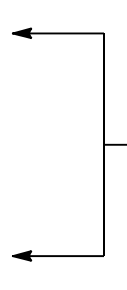

$\mathrm{CF}_{3} \mathrm{CH}_{2} \mathrm{NH}_{2} \mathrm{HCl}$ $\mathrm{NaHCO}_{3}, \mathrm{NaCNBH}_{3}$ $\mathrm{AcOH}, \mathrm{CH}_{2} \mathrm{Cl}_{2}$ $20^{\circ} \mathrm{C}, 3 \mathrm{~h}$

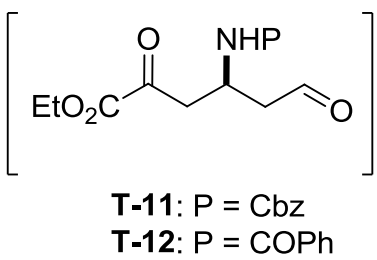

\section{Scheme 4.}

The reductive amination/ring-closure step of T-11 (Cbz protecting group) furnished the expected piperidine amino ester $( \pm)-34$ a regioisomer of $( \pm)$-20. Interestingly, benzoyl-protected amino ester $\mathbf{T - 1 2}$, under the same experimental conditions, gave tetrahydropyridine derivative $( \pm)-35$. It is surmised, that this transformation involves an enamine intermediate (Schemes 4 and 5).

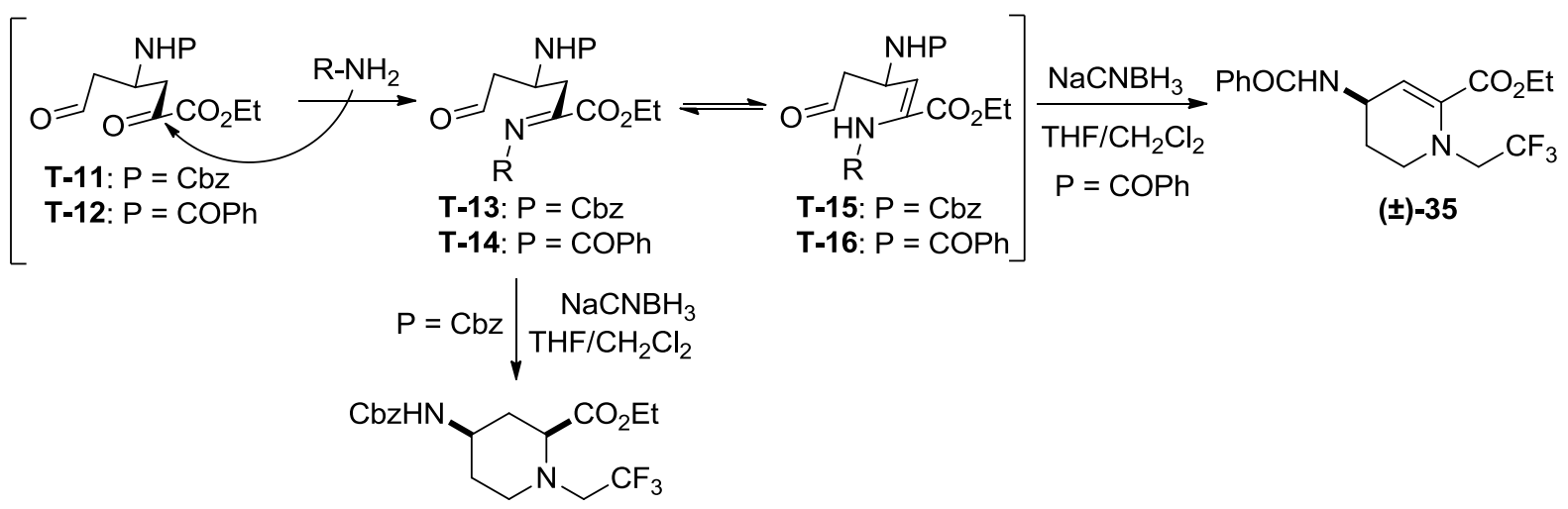

$( \pm)-34$

\section{Scheme 5.}

The synthetic protocol presented above was utilized only for the access of racemates. Our next goal was to prepare enantiomerically pure piperidine $\gamma$-amino esters. Accordingly, we selected and planned to transform enantiopure $\gamma$-lactam (+)-15 as starting material. Since the configurations of the stereogenic centers during the synthetic process were not affected, the enantiopure starting material is expected to produce the corresponding enantiomeric target 
substances. Optically pure (+)-15 was obtained according to our earlier literature procedure, which was based on lactam ring opening through hydrolysis of racemic $( \pm)-15$ catalyzed by Candida antarctica lipase-B (Scheme 6). ${ }^{[11]}$<smiles>O=C1NC2CCC1C2</smiles>

$( \pm)-15$

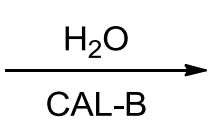

HO<smiles>[R20]C1C=C[C+](C=O)C1</smiles>

$(-)-36$

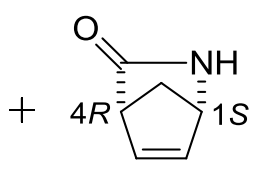

$(+)-15$

Scheme 6.

An illustrative example for the synthesis of a piperidine $\gamma$-amino ester enantiomer is depicted on Scheme 7. Following the reaction sequences described above for the racemates, enatiomerically pure lactam (+)-15 was converted via amino ester (+)-16 and diol (-)-18 into enantiomerically pure $\gamma$-amino ester (+)-20 isolated with an $e e$ of $>99 \%$ (determined by means of HPLC, Chiralpak IA column, Scheme 7).

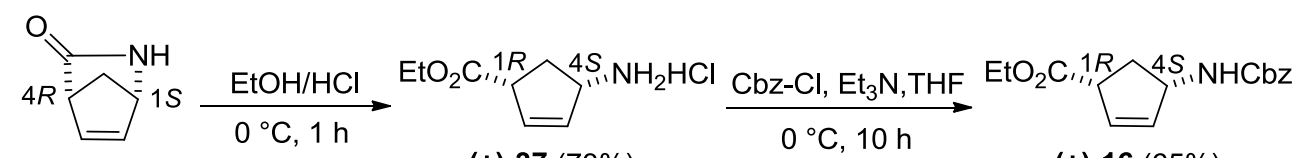
$(+)-15$

$(+)-37(73 \%)$ $(+)-16(65 \%)$

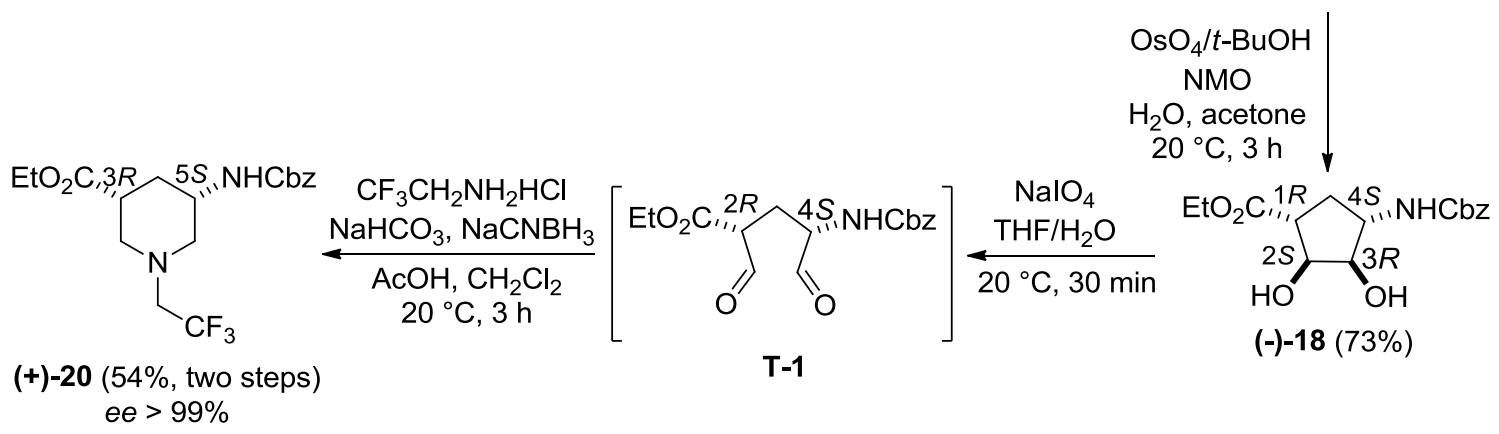

\section{Scheme 7.}

Finally, we decided to further extend the synthetic methodology for the access of novel fluorinecontaining bicyclic lactams. Correspondingly, racemic $N$-Boc-protected Vince lactam ( \pm )-38 was transformed into the corresponding diol derivative $( \pm)-39 .{ }^{[12]}$ 


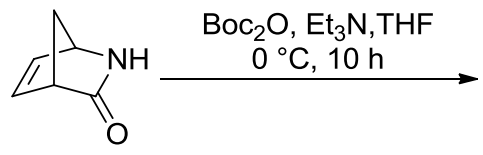

$( \pm)-15$

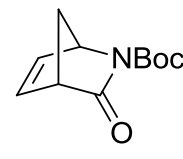

(士)-38 (70\%)

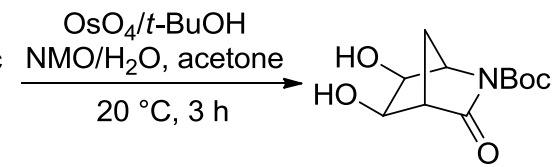

(士)-39 (75\%)

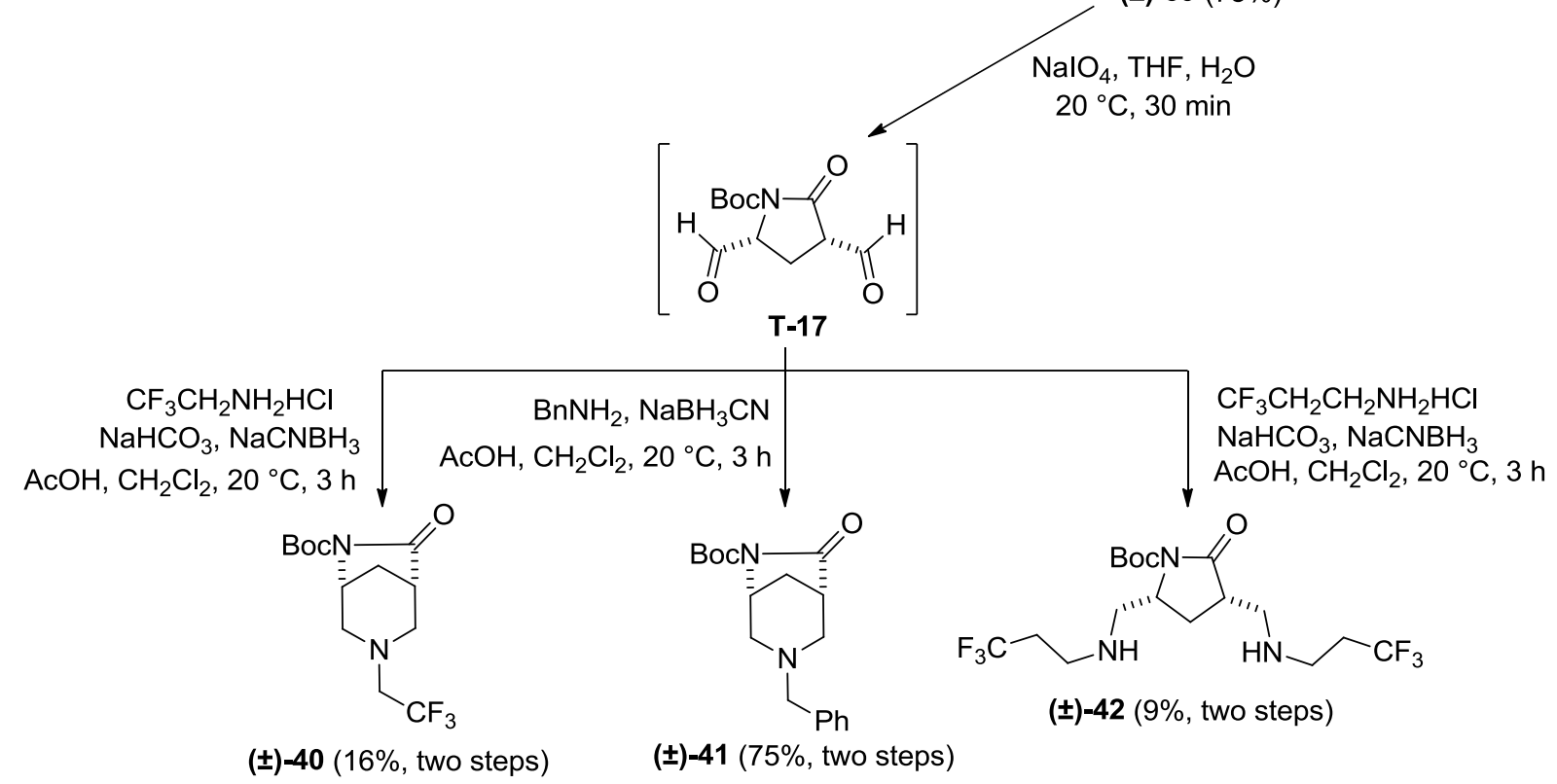

Scheme 8.

Oxidative ring opening of vicinal diol ( \pm )-39 gave diformyl-substituted pyrrolidinone intermediate T-17. Similar to the other dialdehydes, this intermediate is unstable and, therefore, it was immediately subjected to reductive amination. The reaction with trifluoroethylamine or benzylamine led to the desired cyclized azabicyclic compounds ( \pm )-40 and $( \pm)-\mathbf{4 1}$. In contrast, the process with trifluoropropylamine under the same reaction conditions, even at variation of the amount of amine afforded only diamino compounds $( \pm)-42$ (Scheme 8$)$.

\section{Conclusions}

An effective synthetic method for the synthesis of novel fluorine-containing piperidine $\gamma$-amino acid derivatives has been developed. The synthetic strategy, as novel method for the construction of functionalized saturated $N$-heterocycles, was based on oxidative ring opening of unsaturated bicyclic $\gamma$-lactam (Vince-lactam) through its ring olefin bond, followed by reductive ring closure with ring expansion, through reductive amination of the diformyl intermediate performed with various fluoroalkylamines. The novel compounds thus prepared may be regarded as valuable orthogonally-protected $\beta, \gamma$-diaminocarboxylate building scaffolds in synthetic organic and peptide chemistry. Further studies with respect to the transformation of the diformyl intermediates formed in oxidative ring cleavage in view of the synthesis of other 
novel heterocycles as well as preparation of amino acids from the synthetized $N$-protected esters are currently being carried out in our group.

\section{Experimental}

\section{General procedure for $\mathrm{Cbz}$ protection of $\boldsymbol{\gamma}$-amino esters}

To a stirred solution of $\gamma$-amino ester hydrochloride ( $8 \mathrm{mmol}$ ) in THF (50 mL), Et $3 \mathrm{~N}$ (3 equiv) was added at $0{ }^{\circ} \mathrm{C}$ followed by the addition of $\mathrm{Cbz}-\mathrm{Cl}$ ( 1 equiv). The mixture was stirred for 10 $h$ at $20^{\circ} \mathrm{C}$ and then diluted with EtOAc $(80 \mathrm{~mL})$. The organic layer was washed with $\mathrm{H}_{2} \mathrm{O}(3 \times 40$ $\mathrm{mL}$ ), dried over $\mathrm{Na}_{2} \mathrm{SO}_{4}$, filtered and evaporated under reduced pressure. The crude product was purified by means of column chromatography on silica gel ( $n$-hexane/EtOAc) giving the Cbz-protected $\gamma$-amino ester.

\section{General procedure for benzoyl protection of $\boldsymbol{\gamma}$-amino esters}

To a stirred solution of $\gamma$-amino ester hydrochloride $(8 \mathrm{mmol})$ benzoyl chloride ( 1 equiv) was added in toluene $(50 \mathrm{~mL})$, water $(30 \mathrm{~mL})$ and $\mathrm{NaHCO}_{3}\left(3\right.$ equiv) at $0{ }^{\circ} \mathrm{C}$. The reaction mixture was stirred for $1 \mathrm{~h}$ at room temperature. The residue was taken up in EtOAc $(80 \mathrm{~mL})$ and washed with water $(3 \times 40 \mathrm{~mL})$. The organic layer was dried over $\mathrm{Na}_{2} \mathrm{SO}_{4}$ and concentrated under vacuoo. The crude material was purified by column chromatography on silica gel ( $n$ hexane/EtOAc) to give the benzoyl-protected $\gamma$-amino ester.

\section{General procedure for dihydroxylation of $N$-protected amino esters}

To a solution of $N$-protected $\beta$-amino ester ( $5 \mathrm{mmol})$ and NMO (1.2 equiv) in acetone $(30 \mathrm{~mL})$, $0.3 \mathrm{~mL}$ of $2 \% \mathrm{OsO}_{4}$ solution in $t-\mathrm{BuOH}$ was added and the resulting mixture was stirred for 3 $\mathrm{h}$ at room temperature. After the termination of the reaction, $90 \mathrm{~mL}$ of saturated aqueous $\mathrm{Na}_{2} \mathrm{SO}_{3}$ solution was added and the reaction mixture was extracted with $\mathrm{CH}_{2} \mathrm{Cl}_{2}(3 \times 30 \mathrm{~mL})$. The combined organic layers were dried over $\mathrm{Na}_{2} \mathrm{SO}_{4}$, filtered and evaporated under reduced pressure. The crude product was purified by means of column chromatography on silica gel ( $n$ hexane/EtOAc).

General procedure for the synthesis of fluorine-containing $N$-heterocyclic $\gamma$-amino esters and fluorine-containing $N$-heterocyclic $\gamma$-lactams by oxidative ring cleavage followed by ring closure by reductive amination

To a stirred solution of dihydroxylated $\gamma$-amino ester $(2 \mathrm{mmol})$ or dihydroxylated $\gamma$-lactam (2 mmol) $\mathrm{NaIO}_{4}\left(1.5\right.$ equiv) was added in $\mathrm{THF} / \mathrm{H}_{2} \mathrm{O}(25 \mathrm{~mL} / 2 \mathrm{~mL})$. After stirring for $30 \mathrm{~min}$ at 20 
${ }^{\circ} \mathrm{C}$ under an $\mathrm{Ar}$ atmosphere, the reaction was quenched with $\mathrm{H}_{2} \mathrm{O}(40 \mathrm{~mL})$. The mixture was then extracted with $\mathrm{CH}_{2} \mathrm{Cl}_{2}(3 \times 20 \mathrm{~mL})$ and the combined organic layers were dried over $\mathrm{Na}_{2} \mathrm{SO}_{4}$. The resulting solution containing the dialdehyde derivative, concentrated to half of its volume, was used without purification for the next reaction. To the solution of the dialdehyde were added fluorine-containing amine hydrochloride ( 1 equiv) and $\mathrm{NaHCO}_{3}$ (2 equiv) or benzylamine (1 equiv), and the mixture was stirred at $20{ }^{\circ} \mathrm{C}$ for $10 \mathrm{~min}$. After adding $\mathrm{NaCNBH}_{3}$ ( 1 equiv) and $\mathrm{AcOH}$ (2 drops) stirring was continued for another $3 \mathrm{~h}$ at $20{ }^{\circ} \mathrm{C}$. The reaction mixture was diluted with $\mathrm{H}_{2} \mathrm{O}(20 \mathrm{~mL})$ and extracted with $\mathrm{CH}_{2} \mathrm{Cl}_{2}(3 \times 20 \mathrm{~mL})$. The combined organic layers were dried over $\mathrm{Na}_{2} \mathrm{SO}_{4}$ and concentrated under reduced pressure. The residue was purified by column chromatography on silica gel ( $n$-hexane/EtOAc).

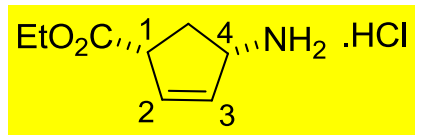

$(1 R, 4 S)$-Ethyl 4-aminocyclopent-2-enecarboxylate hydrochloride (+)-37 ${ }^{[11]}$

White solid, mp: $133-135{ }^{\circ} \mathrm{C}$; yield: $73 \%(1.9 \mathrm{~g}) ;[\alpha]_{\mathrm{D}}{ }^{20}=+178\left(c 0.15, \mathrm{CH}_{2} \mathrm{Cl}_{2}\right)$; ${ }^{1} \mathrm{H}-\mathrm{NMR}$ $\left(500 \mathrm{MHz}, \mathrm{DMSO}_{-} \mathrm{d}_{6}\right): \delta=1.21\left(\mathrm{t}, J=7.05 \mathrm{~Hz}, 3 \mathrm{H}, \mathrm{CH}_{3}\right), 1.93(\mathrm{dt}, J=13.66 \mathrm{~Hz}, J=6.84 \mathrm{~Hz}$, 1H, H-5), 2.55 (dt, $J=13.63 \mathrm{~Hz}, J=8.45 \mathrm{~Hz}, 1 \mathrm{H}, \mathrm{H}-5), 3.65-3.71$ (m, 1H, H-1), 4.08-4.15 (m, 2H, $\mathrm{CH}_{2} \mathrm{CH}_{3}$ ), 4.15-4.21 (m, 1H, H-4), 5.86-5.90 (m, 1H, H-2), 6.06-6.10 (m, 1H, H-3), 8.27 (brs, 3H, NH); ${ }^{13} \mathrm{C}-\mathrm{NMR}$ (126 MHz, DMSO-d 6 ) $\delta=14.6,31.6,49.6,55.7,61.0,130.8,134.9$, 172.4; Anal. Calcd for $\mathrm{C}_{8} \mathrm{H}_{14} \mathrm{ClNO}_{2}$ : C, 50.13; H, 7.36; Cl, 18.50; N, 7.31; found: C, 50.11; H, 7.34; $\mathrm{Cl}, 18.53 ; \mathrm{N}, 7.30$.

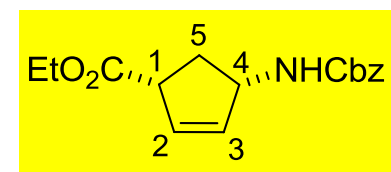

\section{$(1 R, 4 S)$-Ethyl 4-(((benzyloxy)carbonyl)amino)cyclopent-2-enecarboxylate, (+)-16}

White solid, mp: $55-58{ }^{\circ} \mathrm{C}$; yield: $68 \%(1.6 \mathrm{~g}) ; R_{\mathrm{f}}=0.51(n$-hexane/EtOAc $1: 1) ;[\alpha]_{\mathrm{D}}{ }^{20}=+47$ (c $0.15, \mathrm{CH}_{2} \mathrm{Cl}_{2}$ ); ${ }^{1} \mathrm{H}-\mathrm{NMR}\left(500 \mathrm{MHz}, \mathrm{CDCl}_{3}\right): \delta=1.27$ (t, $\left.J=7.00 \mathrm{~Hz}, 3 \mathrm{H}, \mathrm{CH}_{3}\right), 1.90$ (dt, $J$ $=13.97 \mathrm{~Hz}, J=3.59 \mathrm{~Hz}, 1 \mathrm{H}, \mathrm{H}-5), 2.49(\mathrm{dt}, J=14.09 \mathrm{~Hz}, J=8.35 \mathrm{~Hz}, 1 \mathrm{H}, \mathrm{H}-5), 3.43-3.50$ (m, 1H, H-1), 4.09-4.20 (m, 2H, $\left.\mathrm{CH}_{2} \mathrm{CH}_{3}\right), 4.80-4.89$ (m, 1H, H-4), 5.09 (s, 2H, OCH$), 5.21$ (brs, $1 \mathrm{H}, \mathrm{NH}), 5.89$ (s, 2H, H-2 and H-3), 7.27-7.41 (m, 5H, Ar-H); ${ }^{13} \mathrm{C}-\mathrm{NMR}\left(126 \mathrm{MHz}, \mathrm{CDCl}_{3}\right): \delta$ $=14.2,34.5,49.4,56.3,61.1,66.6,128.0,128.1$, 128.5, 131.7, 134.5, 136.6, 155.6, 174.4; Anal. Calcd for $\mathrm{C}_{16} \mathrm{H}_{19} \mathrm{NO}_{4}$ : C, 66.42; H, 6.62; N, 4.84; found: C, 66.40; H, 6.63; N, 4.83. 


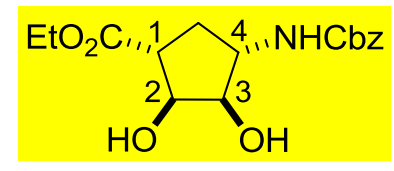

$(1 R, 2 S, 3 R, 4 S)$-Ethyl 4-(((benzyloxy)carbonyl)amino)-2,3-dihydroxycyclopentanecarboxylate, (-)-18

White solid, mp: $74-76{ }^{\circ} \mathrm{C}$; yield: $92 \%(1.4 \mathrm{~g}) ; R_{\mathrm{f}}=0.30(n$-hexane/EtOAc $1: 4) ;[\alpha]_{\mathrm{D}}{ }^{20}=-29(c$ 0.3, $\left.\mathrm{CHCl}_{3}\right) ;{ }^{1} \mathrm{H}-\mathrm{NMR}\left(500 \mathrm{MHz}, \mathrm{DMSO}-\mathrm{d}_{6}\right): \delta=1.18\left(\mathrm{t}, J=7.00 \mathrm{~Hz}, 3 \mathrm{H}, \mathrm{CH}_{3}\right), 1.46(\mathrm{dt}, J=$ $13.07 \mathrm{~Hz}, J=8.92 \mathrm{~Hz}, 1 \mathrm{H}, \mathrm{H}-5), 2.17$ (dt, $J=12.99 \mathrm{~Hz}, J=8.57 \mathrm{~Hz}, 1 \mathrm{H}, \mathrm{H}-5), 2.59-2.67$ (m, 1H, H-1), 3.57-3.63 (m 1H, H-2), 3.67-3.77 (m, 1H, H-4), 3.94-3.99 (m, 1H, H-3), 4.03-4.09 $\left(\mathrm{m}, 2 \mathrm{H}, \mathrm{CH}_{2} \mathrm{CH}_{3}\right), 4.73(\mathrm{~d}, J=5.60,1 \mathrm{H}, \mathrm{OH}), 4.86(\mathrm{~d}, J=5.80,1 \mathrm{H}, \mathrm{OH}), 5.00\left(\mathrm{~s}, 2 \mathrm{H}, \mathrm{OCH}_{2}\right)$, 7.27-7.41 (m, 6H, NH, Ar-H); ${ }^{13} \mathrm{C}-\mathrm{NMR}\left(126 \mathrm{MHz}, \mathrm{DMSO}-\mathrm{d}_{6}\right): \delta=14.6,31.0,47.9,55.9$, 60.5, 65.6, 73.6, 76.0, 128.2, 128.3, 128.8, 137.6, 156.2, 172.3; Anal. Calcd for $\mathrm{C}_{16} \mathrm{H}_{21} \mathrm{NO}_{6}$ : $\mathrm{C}$, 59.43; H, 6.55; N, 4.33; found: C, 59.45; H, 6.54; N, 4.32.

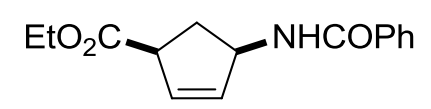

$\left(1 S^{*}, 4 R^{*}\right)$-Ethyl 4-benzamidocyclopent-2-enecarboxylate, $( \pm)-17$

White solid, mp: $73-75{ }^{\circ} \mathrm{C}$; yield: $66 \%(2.3 \mathrm{~g}) ; R_{\mathrm{f}}=0.58$ ( $n$-hexane/EtOAc 1:1); ${ }^{1} \mathrm{H}-\mathrm{NMR}$ $\left(400 \mathrm{MHz}, \mathrm{CDCl}_{3}\right): \delta=1.29\left(\mathrm{t}, J=7.10 \mathrm{~Hz}, 3 \mathrm{H}, \mathrm{CH}_{3}\right), 1.99-2.06(\mathrm{~m}, 1 \mathrm{H}, \mathrm{H}-5), 2.50$ (dt, $J=$ $14.06 \mathrm{~Hz}, J=8.27 \mathrm{~Hz}, 1 \mathrm{H}, \mathrm{H}-5), 3.51-3.58$ (m, $1 \mathrm{H}, \mathrm{H}-1), 4.15-4.23$ (m, 2H, $\mathrm{CH}_{2} \mathrm{CH}_{3}$ ), 5.265.33 (m, 1H, H-4), 5.93-5.98 (m, 1H, H-2), 6.01-6.06 (m, 1H, H-3), 6.92 (br s, 1H, NH), 7.38$7.85(\mathrm{~m}, 5 \mathrm{H}, \mathrm{Ar}-\mathrm{H}) ;{ }^{13} \mathrm{C}-\mathrm{NMR}\left(126 \mathrm{MHz}, \mathrm{CDCl}_{3}\right): \delta=14.2,34.5,49.6,54.5,61.3,127.0,128.5$, 131.4, 132.0, 134.4, 134.6, 166.2, 175.3; Anal. Calcd for $\mathrm{C}_{15} \mathrm{H}_{17} \mathrm{NO}_{3}$ : C, 69.48; H, 6.61; N, 5.40; found: C, 69.49; H, 6.60; N, 5.41.

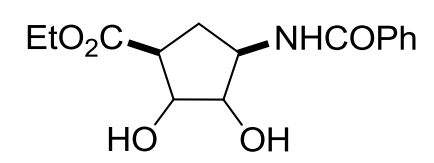

\section{$\left(1 S^{*}, 2 R^{*}, 3 S^{*}, 4 R^{*}\right)$-Ethyl 4-benzamido-2,3-dihydroxycyclopentanecarboxylate, $( \pm)$-19}

White solid, mp: 93-95 ${ }^{\circ} \mathrm{C}$; yield: $97 \%$ (2.5 g); $R_{\mathrm{f}}=0.28$ ( $n$-hexane/EtOAc 1:4). Benzoylprotected $\gamma$ - amino carboxylate dihydroxylation furnished a mixture of diol derivatives which was used in the next step without separation of the components. 


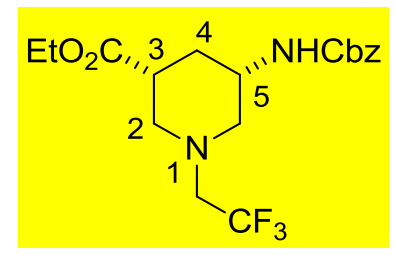

(3R,5S)-Ethyl $\quad 5$-(((benzyloxy)carbonyl)amino)-1-(2,2,2-trifluoroethyl)piperidine-3carboxylate, $(+)-20$

The $e e$ values for the enantiomers were recorded by HPLC [Chiralpak IA column $(4.6 \mathrm{~mm} \times 250$

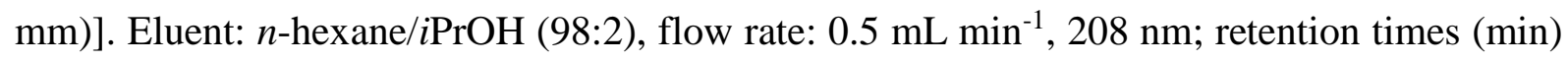
for $(3 R, 5 S)-(+)-17: 66.84$, for $(3 S, 5 R)-(-)-17: 71.84$. White solid, mp: $39-42{ }^{\circ} \mathrm{C}$; yield: $54 \%$ $(396 \mathrm{mg}) ; R_{\mathrm{f}}=0.32\left(n\right.$-hexane/EtOAc 1:1); $[\alpha]_{\mathrm{D}}{ }^{20}=+5(c 0.165, \mathrm{EtOH}), e e=99 \% ;{ }^{1} \mathrm{H}-\mathrm{NMR}$ $\left(400 \mathrm{MHz}, \mathrm{CDCl}_{3}\right): \delta=1.24\left(\mathrm{t}, J=7.10 \mathrm{~Hz}, 3 \mathrm{H}, \mathrm{CH}_{3}\right), 1.43-1.50(\mathrm{~m}, 1 \mathrm{H}, \mathrm{H}-4), 2.16(\mathrm{~d}, J=$ $12.40 \mathrm{~Hz}, 1 \mathrm{H}, \mathrm{H}-4), 2.29-2.40$ (m, 1H, H-3), 2.61-2.73 (m, 2H, H-2), 2.97-3.13 (m, 4H, H-6 and $\mathrm{CH}_{2} \mathrm{CF}_{3}$ ), 3.71-3.82 (m, 1H, H-5), 4.08-4.18 (m, 2H, $\left.\mathrm{CH}_{2}\right), 4.92$ (brs. $\left.1 \mathrm{H}, \mathrm{NH}\right), 5.09$ (s, 2H, $\left.\mathrm{OCH}_{2}\right), 7.29-7.41$ (m, 5H, Ar-H); ${ }^{13} \mathrm{C}-\mathrm{NMR}\left(100 \mathrm{MHz}, \mathrm{CDCl}_{3}\right): \delta=14.5,32.4,40.9,47.3,54.7$, $55.7,58.3\left(\mathrm{q},{ }^{2} J_{\mathrm{C}, \mathrm{F}}=26.7 \mathrm{~Hz}, \mathrm{CCF}_{3}\right), 61.2,67.2,124.5\left({ }^{1} J_{\mathrm{C}, \mathrm{F}}=235.0 \mathrm{~Hz}, \mathrm{CF}_{3}\right), 128.6,128.9$, 136.8, 156.1, 183.2; ${ }^{19} \mathrm{~F}$ NMR (471 MHz, DMSO-d 6 ): $\delta=-67.8(\mathrm{t}, J=10.3 \mathrm{~Hz})$. MS: (ESI) m/z = $389.2(\mathrm{M}+1)$; Anal. Calcd for $\mathrm{C}_{18} \mathrm{H}_{23} \mathrm{~F}_{3} \mathrm{~N}_{2} \mathrm{O}_{4}: \mathrm{C}, 55.67 ; \mathrm{H}, 5.97 ; \mathrm{N}, 7.21$; found: $\mathrm{C}, 55.64 ; \mathrm{H}$, 5.96; N, 7.20.<smiles>CCOC(=O)C1CC(NC(=O)c2ccccc2)CN(CC(F)(F)F)C1</smiles>

$\left(3 S^{*}, 5 R^{*}\right)$-Ethyl 5-benzamido-1-(2,2,2-trifluoroethyl)piperidine-3-carboxylate, $( \pm)-21$

White solid, mp: $67-69{ }^{\circ} \mathrm{C}$; yield: $61 \%(514 \mathrm{mg}) ; R_{\mathrm{f}}=0.45$ ( $n$-hexane/EtOAc 1:1); ${ }^{1} \mathrm{H}-\mathrm{NMR}$ (400 MHz, DMSO-d $\left.\mathrm{d}_{6}\right): \delta=1.19\left(\mathrm{t}, J=7.20 \mathrm{~Hz}, 3 \mathrm{H}, \mathrm{CH}_{3}\right), 1.43-1.56$ (m, 1H, H-4), 2.06-2.13 (m, 1H, H-4), 2.26 (t, $J=10.7 \mathrm{~Hz}, 1 \mathrm{H}, \mathrm{H}-2), 2.37$ (t, $J=11.2 \mathrm{~Hz}, 1 \mathrm{H}, \mathrm{H}-2), 2.63-2.72$ (m, 1H, $\mathrm{H}-3), 3.05$ (dd, $J=10.86 \mathrm{~Hz}, J=3.86 \mathrm{~Hz}, 1 \mathrm{H}, \mathrm{H}-6), 3.13(\mathrm{dd}, J=11.40 \mathrm{~Hz}, J=3.36 \mathrm{~Hz}, 1 \mathrm{H}$, $\mathrm{H}-6), 3.32$ (q, $J=10.22 \mathrm{~Hz}, 2 \mathrm{H}, \mathrm{CH}_{2} \mathrm{CF}_{3}$ ), 3.92-4.02 (m, 1H, H-5), 4.04-4.13 (q, $J=7.04 \mathrm{~Hz}$, $2 \mathrm{H}, \mathrm{CH}_{2}$ ), 7.41-7.86 (m, $\left.5 \mathrm{H}, \mathrm{Ar}-\mathrm{H}\right), 8.27$ (brs. 1H, NH); ${ }^{13} \mathrm{C}-\mathrm{NMR}\left(100 \mathrm{MHz}, \mathrm{DMSO}-\mathrm{d}_{6}\right): \delta=$ $14.9,32.8,41.4,46.3,55.2,57.1\left(\mathrm{q},{ }^{2} J_{\mathrm{C}, \mathrm{F}}=22.0 \mathrm{~Hz}, \mathrm{CCF}_{3}\right), 58.2,61.0,122.8\left({ }^{1} J_{\mathrm{C}, \mathrm{F}}=284.0 \mathrm{~Hz}\right.$, $\left.\mathrm{CF}_{3}\right), 128.1,129.1,132.1,135.3,166.7,173.4 ;{ }^{19} \mathrm{~F}-\mathrm{NMR}\left(376 \mathrm{MHz}, \mathrm{DMSO}-\mathrm{d}_{6}\right): \delta=-67.7(\mathrm{t}, J$ $=10.2 \mathrm{~Hz})$; MS: (ESI) m/z = $359.3(\mathrm{M}+1)$; Anal. Calcd for $\mathrm{C}_{17} \mathrm{H}_{21} \mathrm{~F}_{3} \mathrm{~N}_{2} \mathrm{O}_{3}: \mathrm{C}, 56.98 ; \mathrm{H}, 5.91$; N, 7.82; found: C, 56.96; H, 5.90; N, 7.81. 
<smiles>CCOC(=O)C1CC(NC(=O)OCc2ccccc2)CN(CC(F)F)C1</smiles>

$\left(3 S^{*}, 5 R^{*}\right)$-Ethyl

5-(((benzyloxy)carbonyl)amino)-1-(2,2-difluoroethyl)piperidine-3carboxylate, $( \pm)-22$

Colorless oil; yield: 42\% (191 mg); $R_{\mathrm{f}}=0.74$ ( $n$-hexane/EtOAc 1:1); ${ }^{1} \mathrm{H}-\mathrm{NMR}(400 \mathrm{MHz}$, $\left.\mathrm{CDCl}_{3}\right): \delta=1.22-1.25\left(\mathrm{~m}, 3 \mathrm{H}, \mathrm{CH}_{3}\right), 1.55-1.65$ (m, 2H, H-4), 2.38-2.46 (m, 1H, H-2), $2.52(\mathrm{~d}$, $J=11.44 \mathrm{~Hz}, 1 \mathrm{H}, \mathrm{H}-2), 2.67-2.81$ (m, 4H, H-6, NCH 2 ), 3.02-3.09 (m, 1H, H-3), 3.96-4.03 (m, 1H, H-5), 4.09-4.16 (m, 2H, $\mathrm{CH}_{2} \mathrm{CH}_{3}$ ), 5.05-5.16 (m, 2H, $\mathrm{OCH}_{2}$ ), 5.39 (br.s., 1H, NH), 5.66$5.98\left(\mathrm{tt}, 1 \mathrm{H}, \mathrm{CHF}_{2},{ }^{1} J=55.67 \mathrm{~Hz},{ }^{2} J=4.37 \mathrm{~Hz}\right), 7.29-7.43(\mathrm{~m}, 5 \mathrm{H}, \mathrm{Ar}-\mathrm{H}) ;{ }^{13} \mathrm{C}-\mathrm{NMR}(126$ $\left.\mathrm{MHz}, \mathrm{CDCl}_{3}\right): \delta=14.2,31.0,37.9,45.4,55.6,58.2,58.7\left(\mathrm{t},{ }^{2} J_{\mathrm{C}, \mathrm{F}}=24.6 \mathrm{~Hz}, \mathrm{CCHF}_{2}\right), 60.7$, $66.8,115.3\left(\mathrm{t},{ }^{1} J_{\mathrm{C}, \mathrm{F}}=242.6 \mathrm{~Hz}, \mathrm{CHF}_{2}\right), 128.2,128.3,128.6,136.4,155.4,173.3 ;{ }^{19} \mathrm{~F}-\mathrm{NMR}(471$ $\left.\mathrm{MHz}, \mathrm{CDCl}_{3}\right): \delta=-119.0$; MS: (ESI) $\mathrm{m} / \mathrm{z}=371.3(\mathrm{M}+1)$; Anal. Calcd for $\mathrm{C}_{18} \mathrm{H}_{24} \mathrm{~F}_{2} \mathrm{~N}_{2} \mathrm{O}_{4}: \mathrm{C}$, 58.37; H, 6.53; N, 7.56; found: C, 58.35; H, 6.54; N, 7.55.<smiles>CCOC(=O)C1CC(NC(=O)c2ccccc2)CN(CCF)C1</smiles>

$\left(3 S^{*}, 5 R^{*}\right)$-Ethyl 5-benzamido-1-(2,2-difluoroethyl)piperidine-3-carboxylate, $( \pm)-23$

White solid, mp: $104-107{ }^{\circ} \mathrm{C}$; yield: $63 \%$ (301 mg); $R_{\mathrm{f}}=0.51\left(n\right.$-hexane/EtOAc 1:1); ${ }^{1} \mathrm{H}-\mathrm{NMR}$ $\left(400 \mathrm{MHz}, \mathrm{CDCl}_{3}\right): \delta=1.22\left(\mathrm{t}, J=7.16 \mathrm{~Hz}, 3 \mathrm{H}, \mathrm{CH}_{3}\right), 1.87-1.99(\mathrm{~m}, 1 \mathrm{H}, \mathrm{H}-4), 2.03-2.12(\mathrm{~m}$, 1H, H-4), 2.57-2.66 (m, 1H, H-3), 2.70-2.96 (m, 6H, H-2, H-6, $\mathrm{NCH}_{2}$ ), 4.00-4.20 (m, 2H, $\mathrm{CH}_{2} \mathrm{CH}_{3}$ ), 4.21-4.30 (m, 1H, H-5), 5.69-6.00 (tt, $\left.1 \mathrm{H}, \mathrm{CHF}_{2},{ }^{1} \mathrm{~J}=55.98 \mathrm{~Hz},{ }^{2} \mathrm{~J}=4.15 \mathrm{~Hz}\right), 7.05$ (brs. 1H, NH), 7.36-7.89 (m, 5H, Ar-H); ${ }^{13} \mathrm{C}-\mathrm{NMR}\left(126 \mathrm{MHz}, \mathrm{CDCl}_{3}\right): \delta=14.1,30.7,39.8$, 45.1, 55.1, 58.7, $59.5\left(\mathrm{t},{ }^{2} J_{\mathrm{C}, \mathrm{F}}=25.4 \mathrm{~Hz}, \mathrm{CCHF}_{2}\right), 61.0,115.6\left(\mathrm{t},{ }^{1} J_{\mathrm{C}, \mathrm{F}}=242.6 \mathrm{~Hz}, \mathrm{CHF}_{2}\right), 126.9$, 128.5, 131.5, 134.3, 166.5, 174.4; ${ }^{19} \mathrm{~F}-\mathrm{NMR}\left(471 \mathrm{MHz}, \mathrm{CDCl}_{3}\right): \delta=-118.9 ; \mathrm{MS}:(\mathrm{ESI}) \mathrm{m} / \mathrm{z}=$ $341.3(\mathrm{M}+1)$; Anal. Calcd for $\mathrm{C}_{17} \mathrm{H}_{22} \mathrm{~F}_{2} \mathrm{~N}_{2} \mathrm{O}_{3}$ : C, 59.99; H, 6.51; N, 8.23; found: C, 59.97; $\mathrm{H}$, 6.50; N, 8.24.<smiles>CCOC(=O)C1CC(NC(C)=O)CN(CCF)C1</smiles> 
$\left(3 S^{*}, 5 R^{*}\right)$-Ethyl 5-(((benzyloxy)carbonyl)amino)-1-(2-fluoroethyl)piperidine-3-carboxylate, $( \pm)-24$

White solid, mp: $41-43{ }^{\circ} \mathrm{C}$; yield: $20 \%(126 \mathrm{mg}) ; R_{\mathrm{f}}=0.56(n$-hexane/EtOAc $1: 1) ;{ }^{1} \mathrm{H}-\mathrm{NMR}$ (400 MHz, DMSO-d $): \delta=1.18\left(\mathrm{t}, J=6.8 \mathrm{~Hz}, 3 \mathrm{H}, \mathrm{CH}_{3}\right.$ ), 1.45-1.56 (m, 1H, H-4), 1.82-1.94 (m, 1H, H-4), 2.10-2.21 (m, 1H, H-2), 2.38-2.47 (m, 1H, H-2), 2.54-2.72 (m, 3H, H-3, $\mathrm{CH}_{2-}$ $\mathrm{CH}_{2} \mathrm{~F}$ ), 2.76-2.86 (m, 2H, H-6), 3.67-3.78 (m, 1H, H-5), 3.98-4.14 (m, 2H, $\mathrm{CH}_{2}$ ), 4.38-4.59 (dt, $2 \mathrm{H}, \mathrm{CH}_{2} \mathrm{~F},{ }^{1} \mathrm{~J}=47.85 \mathrm{~Hz},{ }^{2} J=4.94 \mathrm{~Hz}$ ), $5.02\left(\mathrm{~s}, 2 \mathrm{H}, \mathrm{OCH}_{2}\right), 7.10$ (brs. $\left.1 \mathrm{H}, \mathrm{NH}\right), 7.27-7.40$ (m, $5 \mathrm{H}, \mathrm{Ar}-\mathrm{H}) ;{ }^{13} \mathrm{C}-\mathrm{NMR}\left(100 \mathrm{MHz}, \mathrm{DMSO}-\mathrm{d}_{6}\right): \delta=14.9,31.2,39.1,45.9,55.2,58.1\left(\mathrm{~d},{ }^{2} J_{\mathrm{C}, \mathrm{F}}=\right.$ $\left.19.5 \mathrm{~Hz}, \mathrm{CCH}_{2} \mathrm{~F}\right), 59.1,60.7,66.1,82.9\left(\mathrm{~d},{ }^{1} J_{\mathrm{C}, \mathrm{F}}=163.7 \mathrm{~Hz}, \mathrm{CH}_{2} \mathrm{~F}\right), 128.4,128.6,129.2,138.0$, 156.3, 173.8; ${ }^{19} \mathrm{~F}-\mathrm{NMR}\left(376 \mathrm{MHz}, \mathrm{DMSO}-\mathrm{d}_{6}\right): \delta=-216.7$. MS: (ESI) $\mathrm{m} / \mathrm{z}=353.3(\mathrm{M}+1)$; Anal. Calcd for $\mathrm{C}_{18} \mathrm{H}_{25} \mathrm{FN}_{2} \mathrm{O}_{4}$ : C, 61.35; H, 7.15; N, 7.95; found: C, 61.33; H, 7.14; N, 7.94.<smiles>CCOC(=O)C1CC(NC(=O)c2ccccc2)CN(CCF)C1</smiles>

$\left(3 S^{*}, 5 R^{*}\right)$-Ethyl 5-benzamido-1-(2-fluoroethyl)piperidine-3-carboxylate, $( \pm)-25$

White solid, mp: $56-58{ }^{\circ} \mathrm{C}$; yield: $42 \%(134 \mathrm{mg}) ; R_{\mathrm{f}}=0.51$ ( $n$-hexane/EtOAc 1:6); ${ }^{1} \mathrm{H}-\mathrm{NMR}$ $\left(500 \mathrm{MHz}, \mathrm{CDCl}_{3}\right): \delta=1.22\left(\mathrm{t}, J=7.40 \mathrm{~Hz}, 3 \mathrm{H}, \mathrm{CH}_{3}\right), 1.57-1.69(\mathrm{~m}, 1 \mathrm{H}, \mathrm{H}-4), 1.82-1.95$ (m, $1 \mathrm{H}, \mathrm{H}-4), 2.07-2.16$ (m, $1 \mathrm{H}, \mathrm{H}-2), 2.42-2.54$ (m, 1H, H-2), 2.68-2.94 (m, 5H, H-3, $\mathrm{CH}_{2}-\mathrm{CH}_{2} \mathrm{~F}$, H-6), 4.01-4.21 (m, 2H, CH$), 4.23-4.31$ (m, 1H, H-5), 4.46-4.69 (m, 2H, $\left.\mathrm{CH}_{2} \mathrm{~F}\right), 6.97$ (brs. 1H, $\mathrm{NH}), 7.40-7.84$ (m, 5H, Ar-H); ${ }^{13} \mathrm{C}-\mathrm{NMR}\left(126 \mathrm{MHz}, \mathrm{CDCl}_{3}\right): \delta=14.1,30.8,39.9,45.3,54.9$, $57.0\left(\mathrm{~d},{ }^{2} J_{\mathrm{C}, \mathrm{F}}=20.0 \mathrm{~Hz}, \mathrm{CCH}_{2} \mathrm{~F}\right), 58.4,60.9,82.1\left(\mathrm{~d},{ }^{1} J_{\mathrm{C}, \mathrm{F}}=140.0 \mathrm{~Hz}, \mathrm{CH}_{2} \mathrm{~F}\right), 126.9,128.5$, 131.4, 134.5, 166.5, 174.4; ${ }^{19} \mathrm{~F}-\mathrm{NMR}$ (471 MHz, DMSO-d 6 ): $\delta=-218.2$; MS: (ESI) $\mathrm{m} / \mathrm{z}=$ $322.37(\mathrm{M}+1)$; Anal. Calcd for $\mathrm{C}_{17} \mathrm{H}_{23} \mathrm{FN}_{2} \mathrm{O}_{3}$ : C, 63.34; H, 7.19; N, 8.69; found: $\mathrm{C}, 63.32 ; \mathrm{H}$, $7.18 ; \mathrm{N}, 8.68$.

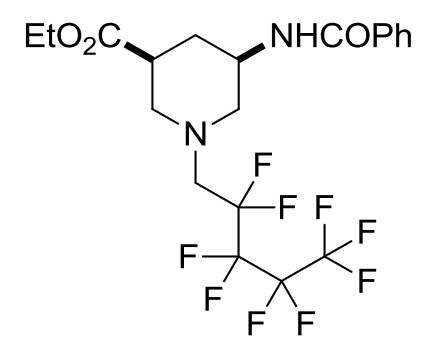

$\left(3 S^{*}, 5 R^{*}\right)$-Ethyl $\quad 5$-benzamido-1-(2,2,3,3,4,4,5,5,5-nonafluoropentyl)piperidine-3-carboxylate, $( \pm)-26$ 
Colorless oil; yield: $17 \%(176 \mathrm{mg}) ; R_{\mathrm{f}}=0.31$ (n-hexane/EtOAc 2:1); ${ }^{1} \mathrm{H}-\mathrm{NMR}(500 \mathrm{MHz}$, $\left.\mathrm{CDCl}_{3}\right): \delta=1.23\left(\mathrm{t}, J=7.30 \mathrm{~Hz}, 3 \mathrm{H}, \mathrm{CH}_{3}\right), 1.63(\mathrm{td}, J=13.26 \mathrm{~Hz}, J=2.98 \mathrm{~Hz}, 1 \mathrm{H}, \mathrm{H}-4), 2.32-$ 2.38 (m, 1H, H-4), 2.57-2.64 (m, 1H, H-3), 2.72-3.27 (m, 6H, H-2, $\mathrm{CH}_{2} \mathrm{CF}_{2}, \mathrm{H}-6$ ), 4.06-4.14 (m, 2H, $\mathrm{CH}_{2}$ ), 4.41-4.48 (m, 1H, H-5), 6.93 (brs. 1H, NH), 7.40-7.82 (m, 5H, Ar-H); ${ }^{13} \mathrm{C}-\mathrm{NMR}$ $\left(126 \mathrm{MHz}, \mathrm{CDCl}_{3}\right): \delta=14.1,30.6,38.1,44.4,56.1,57.0\left(\mathrm{t},{ }^{2} J_{\mathrm{C}, \mathrm{F}}=23.3 \mathrm{~Hz}, \mathrm{CCHF}_{2}\right), 58.6$, 60.8, 117.3-124.4 (m, 4C, $\left.\left(\mathrm{CF}_{2}\right)_{3} \mathrm{CF}_{3}\right)$ 126.8, 128.6, 131.6, 134.2, 166.3, 173.0; ${ }^{19} \mathrm{~F}-\mathrm{NMR}(471$ MHz, DMSO-d 6 ): $\delta=-80.9$ (t, $J=9.58 \mathrm{~Hz}$ ), -115.8, -123.4, -126.1; MS: (ESI) m/z = 509.41 $(\mathrm{M}+1)$; Anal. Calcd for $\mathrm{C}_{20} \mathrm{H}_{21} \mathrm{~F}_{9} \mathrm{~N}_{2} \mathrm{O}_{3}: \mathrm{C}, 47.25 ; \mathrm{H}, 4.16 ; \mathrm{N}, 5.51$; found: $\mathrm{C}, 47.24 ; \mathrm{H}, 4.15 ; \mathrm{N}$, 5.50 .<smiles>CCOC(=O)C1CC(NC(=O)c2ccccc2)CN(CCC(F)(F)F)C1</smiles>

$\left(3 S^{*}, 5 R^{*}\right)$-Ethyl 5-benzamido-1-(3,3,3-trifluoropropyl)piperidine-3-carboxylate, $( \pm)-27$

White solid, mp: $113-115{ }^{\circ} \mathrm{C}$; yield: $24 \%$ (128 mg); $R_{\mathrm{f}}=0.52(n$-hexane/EtOAc $1: 1) ;{ }^{1} \mathrm{H}-\mathrm{NMR}$ (400MHz, DMSO-d 6 ): $\delta=1.19$ (t, $\left.J=6.8 \mathrm{~Hz}, 3 \mathrm{H}, \mathrm{CH}_{3}\right), 1.43-1.56$ (m, 1H, H-4), 1.85-2.04 (m, 2H, H-2), 2.05-2.13 (m, 1H, H-4), 2.36-2.49 (m, 2H, $\mathrm{CH}_{2} \mathrm{CF}_{3}$ ), 2.57-2.71 (m, 3H, H-3, $\mathrm{NCH}_{2}$ ), 2.94-3.09 (m, 2H, H-6), 3.92-4.02 (m, 1H, H-5), 4.08 (q, J = 7.07 Hz, 2H, $\mathrm{CH}_{2}$ ), 7.41-7.88 (m, $5 \mathrm{H}, \mathrm{Ar}-\mathrm{H}), 8.26$ (brs. $1 \mathrm{H}, \mathrm{NH}) ;{ }^{13} \mathrm{C}-\mathrm{NMR}\left(126 \mathrm{MHz}, \mathrm{DMSO}-\mathrm{d}_{6}\right): \delta=14.5,30.9$ (q, ${ }^{2} J_{\mathrm{C}, \mathrm{F}}=26.5$ $\left.\mathrm{Hz}, \mathrm{CCF}_{3}\right) 32.8,41.0,46.0,50.3,54.5,57.5,60.6,127.7$ (q, ${ }^{1} J_{\mathrm{C}, \mathrm{F}}=277.20 \mathrm{~Hz}, \mathrm{CF}_{3}$ ), 127.8, 128.7, 131.7, $134.8166 .2,173.1 ;{ }^{19} \mathrm{~F}-\mathrm{NMR}$ (471 MHz, DMSO-d 6 ): $\delta=-63.5$ (t, $\left.J=11.70 \mathrm{~Hz}\right)$. MS: (ESI) $\mathrm{m} / \mathrm{z}=373.4(\mathrm{M}+1)$; Anal. Calcd for $\mathrm{C}_{18} \mathrm{H}_{23} \mathrm{~F}_{3} \mathrm{~N}_{2} \mathrm{O}_{3}$ : C, 58.06; H, 6.23; N, 7.52; found: C, 58.04; H, 6.21; N, 7.50.<smiles>CCOC(=O)C1CC(NC(C)=O)CN(Cc2ccccc2)C1</smiles>

$\left(3 S^{*}, 5 R^{*}\right)$-Ethyl 1-benzyl-5-(((benzyloxy)carbonyl)amino)piperidine-3-carboxylate, $( \pm)$ 28

Yellow solid, mp: $74-77{ }^{\circ} \mathrm{C}$; yield: $26 \%(127 \mathrm{mg}) ; R_{\mathrm{f}}=0.30$ ( $n$-hexane/EtOAc $\left.2: 1\right) ;{ }^{1} \mathrm{H}-\mathrm{NMR}$ $\left(500 \mathrm{MHz}, \mathrm{CDCl}_{3}\right): \delta=1.22\left(\mathrm{t}, J=7.2 \mathrm{~Hz}, 3 \mathrm{H}, \mathrm{CH}_{3}\right), 1.54-1.64(\mathrm{~m}, 1 \mathrm{H}, \mathrm{H}-4), 2.09-2.19$ (m, 
2H, H-2), 2.21-2.29 (m, 1H, H-4), 2.66-2.77 (m, 2H, H-6), 2.96-3.05 (m, 1H, H-3), 3.44-3.54 (m, 2H, NCH$)_{2}$, 3.96-4.01 (m, 1H, H-5), 4.04-4.14 (m, 2H, $\left.\mathrm{CH}_{2} \mathrm{CH}_{3}\right)$, 5.02-5.16 (m, 2H, $\mathrm{OCH}_{2}$ ), 4.56 (brs. $1 \mathrm{H}, \mathrm{NH}), 7.22-7.40(\mathrm{~m}, 10 \mathrm{H}, \mathrm{Ar}-\mathrm{H}) ;{ }^{13} \mathrm{C}-\mathrm{NMR}\left(126 \mathrm{MHz}, \mathrm{CDCl}_{3}\right): \delta=14.2,31.6$, $37.9,45.5,54.9,57.3,60.5,62.8,66.7,127.3,127.8,128.0,128.2,128.3,128.4,128.5,128.6$, 128.8, 128.9, 136.5, 137.6, 155.4, 173.7; MS: (ESI) m/z = $397.2(\mathrm{M}+1)$; Anal. Calcd for $\mathrm{C}_{23} \mathrm{H}_{28} \mathrm{~N}_{2} \mathrm{O}_{4}$ : C, 69.67; H, 7.12; N, 7.07; found: C, 69.65; H, 7.11; N, 7.08.<smiles>CCOC(=O)C1CC(NC(=O)c2ccccc2)CN(Cc2ccccc2)C1</smiles>

$\left(3 S^{*}, 5 R^{*}\right)$-Ethyl 5-benzamido-1-benzylpiperidine-3-carboxylate, $( \pm)-29$

White solid, mp: $106-109{ }^{\circ} \mathrm{C}$; yield: $21 \%(110 \mathrm{mg}) ; R_{\mathrm{f}}=0.51(n$-hexane/EtOAc $1: 1) ;{ }^{1} \mathrm{H}-\mathrm{NMR}$ (400MHz, DMSO-d 6$): \delta=1.16\left(\mathrm{t}, J=7.2 \mathrm{~Hz}, 3 \mathrm{H}, \mathrm{CH}_{3}\right), 1.42-1.55(\mathrm{~m}, 1 \mathrm{H}, \mathrm{H}-4), 1.86(\mathrm{t}, J=$ $10.58 \mathrm{~Hz}, 1 \mathrm{H}, \mathrm{H}-2), 1.94$ (t, $J=11.12 \mathrm{~Hz}, 1 \mathrm{H}, \mathrm{H}-2), 2.05-2.13$ (m, 1H, H-4), 2.60-2.72 (m, 1H, $\mathrm{H}-3), 2.89-3.04$ (m, 2H, H-6), 3.56 (s, 2H, $\mathrm{NCH}_{2}$ ), 3.94-4.10 (m, 3H, H-5, $\left.\mathrm{CH}_{2} \mathrm{CH}_{3}\right), 7.21-7.87$ (m, 10H, Ar-H), 8.25 (brs. 1H, NH); ${ }^{13} \mathrm{C}-\mathrm{NMR}\left(126 \mathrm{MHz}, \mathrm{DMSO}-\mathrm{d}_{6}\right): \delta=14.5,32.9,41.1$, 46.1, 54.6, 57.7, 60.6, 62.1, 127.5, 127.7, 128.7, 129.2, 131.7, 134.8, 138.4, 166.2, 173.2; MS: (ESI) $\mathrm{m} / \mathrm{z}=367.2(\mathrm{M}+1)$; Anal. Calcd for $\mathrm{C}_{22} \mathrm{H}_{26} \mathrm{~N}_{2} \mathrm{O}_{3}$ : C, 72.11; H, 7.15; N, 7.64; found: $\mathrm{C}$, 72.09; H, 7.14; N, 7.65.

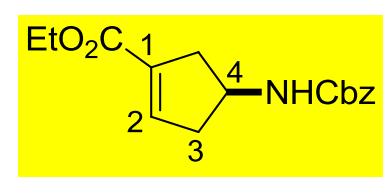

\section{$\left(S^{*}\right)$-Ethyl 4-(((benzyloxy)carbonyl)amino)cyclopent-1-enecarboxylate, $( \pm)$-30}

Colorless oil; yield: $30 \%(0.5 \mathrm{~g}) ; R_{\mathrm{f}}=0.46$ ( $n$-hexane/acetone $\left.2: 1\right) ;{ }^{1} \mathrm{H}-\mathrm{NMR}(500 \mathrm{MHz}$, $\left.\mathrm{CDCl}_{3}\right): \delta=1.28\left(\mathrm{t}, J=7.07 \mathrm{~Hz}, 3 \mathrm{H}, \mathrm{CH}_{3}\right), 2.36-2.50(\mathrm{~m}, 2 \mathrm{H}, \mathrm{H}-3, \mathrm{H}-5), 2.87-3.03(\mathrm{~m}, 2 \mathrm{H}, \mathrm{H}-$ 3, H-5), 4.19 (q, J=7.13 Hz, 2H, $\mathrm{CH}_{2} \mathrm{CH}_{3}$ ), 4.38-4.47 (m, 1H, H-4), 4.98 (br s, 1H, NH), 5.08 (s, 2H, $\left.\mathrm{OCH}_{2}\right), 6.67-6.73$ (m, 1H, H-2), 7.29-7.39 (m, 5H, Ar-H); ${ }^{13} \mathrm{C}-\mathrm{NMR}\left(126 \mathrm{MHz}, \mathrm{CDCl}_{3}\right)$ : $\delta=14.3,39.1,41.1,50.7,60.4,60.7,128.1,128.2,128.6,134.8,136.4,140.7,155.8,164.2$; Anal. Calcd for $\mathrm{C}_{16} \mathrm{H}_{19} \mathrm{NO}_{4}$ : C, 66.42; H, 6.62; N, 4.84; found: C, 66.40; H, 6.63; N, 4.86. 


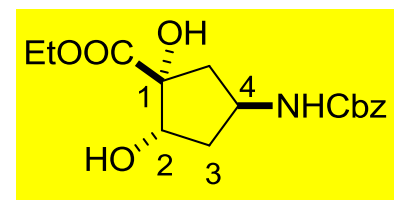

$\left(1 R^{*}, 2 S^{*}, 4 S^{*}\right)$-Ethyl 4-(((benzyloxy)carbonyl)amino)-1,2-dihydroxycyclopentanecarboxylate, $( \pm)-32$

White solid, mp: $78-80{ }^{\circ} \mathrm{C}$; yield: $65 \%$ (450 mg); $R_{\mathrm{f}}=0.47$ ( $n$-hexane/EtOAc 1:2); ${ }^{1} \mathrm{H}-\mathrm{NMR}$ $\left(500 \mathrm{MHz}, \mathrm{DMSO}-\mathrm{d}_{6}\right): \delta=1.19\left(\mathrm{t}, J=7.03 \mathrm{~Hz}, 3 \mathrm{H}, \mathrm{CH}_{3}\right), 1.72-1.79$ (m, 1H, H-3), 1.85-1.93 (m, 2H, H-3, H-5), 2.03 (dd, $J=13.23 \mathrm{~Hz}, J=7.78 \mathrm{~Hz}, 1 \mathrm{H}, \mathrm{H}-5), 4.03-4.14$ (m, 3H, $\mathrm{CH}_{2} \mathrm{CH}_{3}$, $\mathrm{H}-4), 4.26-4.34$ (m, 1H, H-2), 4.87 (s, 1H, OH), 4.93 (d, J=7.1, 1H, OH), 4.99 (s, 2H, $\mathrm{OCH}_{2}$ ), 7.03-7.38 (m, 5H, Ar-H), 7.42 (brs. $1 \mathrm{H}, \mathrm{NH}) ;{ }^{13} \mathrm{C}-\mathrm{NMR}\left(126 \mathrm{MHz}, \mathrm{DMSO}-\mathrm{d}_{6}\right): \delta=14.6,38.6$, $42.8,48.0,60.9,65.6,75.4,81.2,128.3,128.8,137.7,156.0,174.6$; Anal. Calcd for $\mathrm{C}_{16} \mathrm{H}_{21} \mathrm{NO}_{6}$ : C, 59.43; H, 6.55; N, 4.33; found: C, 59.41; H, 6.56; N, 4.30 .

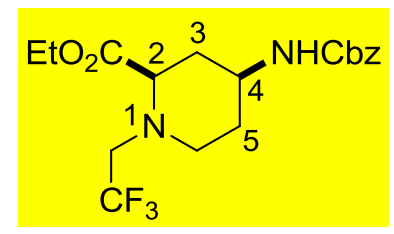

$\left(2 R^{*}, 4 S^{*}\right)$-Ethyl $\quad 4$-(((benzyloxy)carbonyl)amino)-1-(2,2,2-trifluoroethyl)piperidine-2carboxylate, $( \pm)-33$

White solid, mp: $83-85{ }^{\circ} \mathrm{C}$; yield: $25 \%(140 \mathrm{mg}) ; R_{\mathrm{f}}=0.65$ ( $n$-hexane/EtOAc 4:1); ${ }^{1} \mathrm{H}-\mathrm{NMR}$ (500 MHz, DMSO-d 6 ): $\delta=1.24\left(\mathrm{t}, J=7.16 \mathrm{~Hz}, 3 \mathrm{H}, \mathrm{CH}_{3}\right), 1.54-1.65$ (m, 1H, H-5), 1.82-1.98 (m, 2H, H-5, H-3), 2.12-2.22 (m, 1H, H-3), 2.62-2.72 (m, 1H, H-6), 3.10-3.26 (m, 3H, H-6, $\mathrm{CH}_{2} \mathrm{CF}_{3}$ ), 3.40-3.47 (m, 1H, H-2). 3.69-3.80 (m, $\left.1 \mathrm{H}, \mathrm{H}-4\right), 4.08-4.22$ (m, $2 \mathrm{H}, \mathrm{CH}_{2} \mathrm{CH}_{3}$ ), 4.84 (brs. 1H, NH), 5.02-5.13 (m, 2H, $\left.\mathrm{OCH}_{2}\right), 7.29-7.40$ (m, 5H, Ar-H); ${ }^{13} \mathrm{C}-\mathrm{NMR}(126 \mathrm{MHz}$, DMSO-d $\left.{ }_{6}\right): \delta=14.1,31.1,34.6,45.7,48.8,55.6\left(\mathrm{q},{ }^{2} J_{\mathrm{C}, \mathrm{F}}=30.5 \mathrm{~Hz}, \mathrm{CCF}_{3}\right), 61.1,61.6,66.7$, $125.5\left(\mathrm{q},{ }^{1} J_{\mathrm{C}, \mathrm{F}}=284.7 \mathrm{~Hz}, \mathrm{CF}_{3}\right), 128.2,128.6,136.3,155.5,172.8 ;{ }^{19} \mathrm{~F}-\mathrm{NMR}$ (471 MHz, DMSO$\left.\mathrm{d}_{6}\right): \delta=-69.3$; MS: (ESI) $\mathrm{m} / \mathrm{z}=389.56(\mathrm{M}+1)$; Anal. Calcd for $\mathrm{C}_{18} \mathrm{H}_{23} \mathrm{~F}_{3} \mathrm{~N}_{2} \mathrm{O}_{4}: \mathrm{C}, 55.67 ; \mathrm{H}$, 5.97; N, 7.21; found: C, 55.64; H, 5.98; N, 7.20.

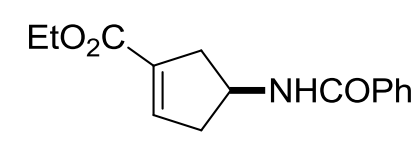

$\left(S^{*}\right)$-Ethyl 4-benzamidocyclopent-1-enecarboxylate, $( \pm)-31$

Colorless oil; yield: $33 \%$ (363 mg); $R_{\mathrm{f}}=0.19$ ( $n$-hexane/acetone 4:1); ${ }^{1} \mathrm{H}-\mathrm{NMR}(400 \mathrm{MHz}$, DMSO-d $\left.{ }_{6}\right): \delta=1.23\left(\mathrm{t}, J=7.20 \mathrm{~Hz}, 3 \mathrm{H}, \mathrm{CH}_{3}\right), 2.52-2.64$ (m, 2H, H-3, H-5), 2.83-2.96 (m, 2H, 
H-3, H-5), 4.14 (q, J = 7.10 Hz, 2H, $\mathrm{CH}_{2} \mathrm{CH}_{3}$ ), 4.56-4.66 (m, 1H, H-4), 6.69-6.74 (m, 1H, H2), 7.40-7.91 (m, 5H, Ar-H), 8.54 (brs. $1 \mathrm{H}, \mathrm{NH}) ;{ }^{13} \mathrm{C}-\mathrm{NMR}\left(126 \mathrm{MHz}, \mathrm{CDCl}_{3}\right): \delta=14.7,38.5$, 40.6, 49.3, 60.3, 127.8, 128.6, 131.6, 134.6, 134.9, 142.1, 164.5, 166.5; Anal. Calcd for $\mathrm{C}_{15} \mathrm{H}_{17} \mathrm{NO}_{3}$ : C, 69.48; H, 6.61; N, 5.40; found: C, 69.51; H, 6.59; N, 5.38.

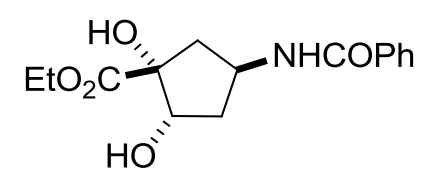

$\left(1 R^{*}, 2 S^{*}, 4 S^{*}\right)$-Ethyl 4-benzamido-1,2-dihydroxycyclopentanecarboxylate, $( \pm)$-33

White solid, mp: $87-89{ }^{\circ} \mathrm{C}$; yield: $59 \%$ (248 mg); $R_{\mathrm{f}}=0.16$ ( $n$-hexane/EtOAc 1:4); ${ }^{1} \mathrm{H}-\mathrm{NMR}$ (400 MHz, DMSO-d $\left.)_{6}\right): \delta=1.21\left(\mathrm{t}, J=7.20 \mathrm{~Hz}, 3 \mathrm{H}, \mathrm{CH}_{3}\right.$ ), 1.86-2.02 (m, 2H, H-5), 2.03-2.12 (m, 2H, H-3), 4.12 (q, J = 7.20 Hz 2H, $\left.\mathrm{CH}_{2} \mathrm{CH}_{3}\right), 4.37-4.56(\mathrm{~m}, 1 \mathrm{H}, \mathrm{H}-2), 4.46-4.55$ (m, 1H, H-4), 4.85 (s, 1H, OH), 4.90 (d, J= 7.1, 1H, OH), 7.41-7.88 (m, 5H, Ar-H), 8.46 (brs. 1H, NH); ${ }^{13} \mathrm{C}-\mathrm{NMR}\left(126 \mathrm{MHz}, \mathrm{DMSO}-\mathrm{d}_{6}\right): \delta=14.6,38.4,42.5,46.7,60.9,75.7,81.4,127.7,128.6$, 131.5, 135.0, 166.1, 174.7; Anal. Calcd for $\mathrm{C}_{15} \mathrm{H}_{19} \mathrm{NO}_{5}$ : C, 61.42; H, 6.53; N, 4.78; found: C, $61.39 ; \mathrm{H}, 6.54 ; \mathrm{N}, 4.76$.

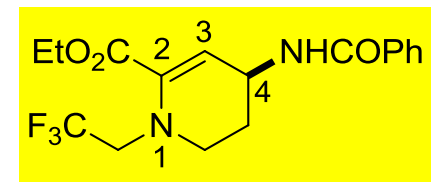

( $\left.S^{*}\right)$-Ethyl 4-benzamido-1-(2,2,2-trifluoroethyl)-1,4,5,6-tetrahydropyridine-2-carboxylate, $( \pm)-35$

White solid, mp: $83-85{ }^{\circ} \mathrm{C}$; yield: $23 \%(143 \mathrm{mg}) ; R_{\mathrm{f}}=0.21(n$-hexane/EtOAc $3: 1)$; ${ }^{1} \mathrm{H}-\mathrm{NMR}$ (400 MHz, DMSO-d $\left.\mathrm{d}_{6}\right): \delta=1.22\left(\mathrm{t}, J=7.30 \mathrm{~Hz}, 3 \mathrm{H}, \mathrm{CH}_{3}\right), 1.74-1.85$ (m, 1H, H-5), 1.86-1.95 (m, 1H, H-5), 3.29-3.33 (m, 2H, H-6), 3.91-4.10 (m, 2H, $\left.\mathrm{CH}_{2} \mathrm{CF}_{3}\right) .4 .17$ (q, J = 7.01 Hz, 2H, $\left.\mathrm{CH}_{2} \mathrm{CH}_{3}\right), 4.60-4.68(\mathrm{~m}, 1 \mathrm{H}, \mathrm{H}-4), 5.64(\mathrm{~d}, J=3.56 \mathrm{~Hz}, 1 \mathrm{H}, \mathrm{H}-3), 7.31-7.91(\mathrm{~m}, 5 \mathrm{H}, \mathrm{Ar}-\mathrm{H})$, 8.49 (brs. $1 \mathrm{H}, \mathrm{NH}) ;{ }^{13} \mathrm{C}-\mathrm{NMR}\left(100 \mathrm{MHz}, \mathrm{DMSO}-\mathrm{d}_{6}\right): \delta=14.8,28.8,43.1,48.0,51.9$ (q, ${ }^{2} J_{\mathrm{C}, \mathrm{F}}$ $\left.=33.3 \mathrm{~Hz}, \mathrm{CCF}_{3}\right), 61.7,114.2,126.5\left(\mathrm{q},{ }^{1} J_{\mathrm{C}, \mathrm{F}}=273.0 \mathrm{~Hz}, \mathrm{CF}_{3}\right), 128.3,129.0,132.0,135.2$, 137.9, 164.6, 166.6; ${ }^{19} \mathrm{~F}-\mathrm{NMR}\left(376 \mathrm{MHz}, \mathrm{DMSO}_{-} \mathrm{d}_{6}\right): \delta=-68.5(\mathrm{t}, J=9.5 \mathrm{~Hz})$; MS: (ESI) m/z = $379.41(\mathrm{M}+23)$; Anal. Calcd for $\mathrm{C}_{17} \mathrm{H}_{19} \mathrm{~F}_{3} \mathrm{~N}_{2} \mathrm{O}_{3}$ : C, 57.30; H, 5.37; N, 7.86; found: C, 57.27; H, 5.39; N, 7.84.

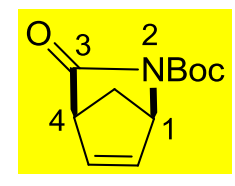


$\left(1 R^{*}, 4 S^{*}\right)-t$-Butyl 3-oxo-2-azabicyclo[2.2.1] hept-5-ene-2-carboxylate, $( \pm)-38^{[11]}$

White solid, mp: $56-58{ }^{\circ} \mathrm{C}$; yield: $70 \%(1.3 \mathrm{~g}) ; R_{\mathrm{f}}=0.40(n$-hexane/EtOAc $2: 1) ;{ }^{1} \mathrm{H}-\mathrm{NMR}(500$ $\left.\mathrm{MHz}, \mathrm{CDCl}_{3}\right): \delta=1.50\left(\mathrm{~s}, 9 \mathrm{H}, \mathrm{CH}_{3}\right), 2.12-2.18\left(\mathrm{~m}, 1 \mathrm{H}, \mathrm{CH}_{2}\right), 2.32-2.38\left(\mathrm{~m}, 1 \mathrm{H}, \mathrm{CH}_{2}\right), 3.36-$ 3.42 (m, 1H, H-4), 4.93-4.99 (m, 1H, H-1), 6.64-6.68 (m, 1H, H-5), 6.88-6.92 (m, 1H, H-6); ${ }^{13} \mathrm{C}-\mathrm{NMR}\left(126 \mathrm{MHz}, \mathrm{CDCl}_{3}\right): \delta=28.1,54.5,55.0,62.4,82.6,138.2,140.0,150.4,176.3$; Anal. Calcd for $\mathrm{C}_{11} \mathrm{H}_{15} \mathrm{NO}_{3}$ : C, 63.14; H, 7.23; N, 6.69; found: C, 63.12; H, 7.21; N, 6.72.

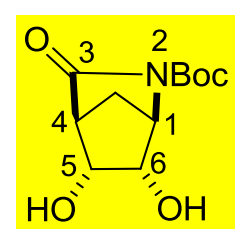

$\left(1 R^{*}, 4 S^{*}, 5 R^{*}, 6 S^{*}\right)-t$-Butyl 5,6-dihydroxy-3-oxo-2-azabicyclo[2.2.1]heptane-2-carboxylate, $( \pm)-39^{[12]}$

White solid, mp: $155-157{ }^{\circ} \mathrm{C}$; yield: $75 \%(0.9 \mathrm{~g}) ; R_{\mathrm{f}}=0.27$ ( $n$-hexane/EtOAc 1:2); ${ }^{1} \mathrm{H}-\mathrm{NMR}$ (500 MHz, DMSO-d 6 ): $\delta=1.52\left(\mathrm{~s}, 9 \mathrm{H}, \mathrm{CH}_{3}\right), 1.95-2.04\left(\mathrm{~m}, 1 \mathrm{H}, \mathrm{CH}_{2}\right), 2.06-2.12\left(\mathrm{~m}, 1 \mathrm{H}, \mathrm{CH}_{2}\right)$, 2.78-2.82 (m, 1H, H-4), 4.09-4.17 (m, 1H, H-5), 4.24-4.30 (m, 1H, H-6), 4.32-4.38 (m, 1H, H1); ${ }^{13} \mathrm{C}-\mathrm{NMR}$ (126 MHz, DMSO-d 6 ): $\delta=28.1$, 31.7, 53.9, 62.4, 68.2, 70.5, 82.4, 149.1, 172.6; Anal. Calcd for $\mathrm{C}_{11} \mathrm{H}_{17} \mathrm{NO}_{5}$ : C, 54.31; H, 7.04; N, 5.76; found: C, 54.35; H, 7.02; N, 5.77.

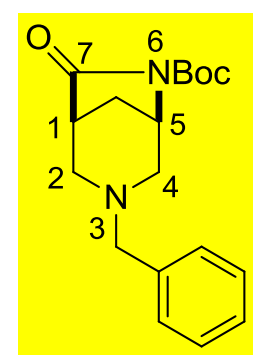

$\left(1 S^{*}, 5 R^{*}\right)$-t-Butyl 3-benzyl-7-oxo-3,6-diazabicyclo[3.2.1] octane-6-carboxylate, $( \pm)-40$

Yellow solid, mp: $84-86{ }^{\circ} \mathrm{C}$; yield: $75 \%(590 \mathrm{mg}) ; R_{\mathrm{f}}=0.40$ ( $n$-hexane/EtOAc 4:1); ${ }^{1} \mathrm{H}-\mathrm{NMR}$ (500MHz, DMSO-d 6$): \delta=1.40$ (s, 9H, $\left.\mathrm{CH}_{3}\right), 1.57-1.61$ (m, 1H, H-8), 2.09-2.20 (m, 2H, H-2), 2.37 (d, $J=10.20 \mathrm{~Hz}, 1 \mathrm{H}, \mathrm{H}-8), 2.48-2.50$ (m, $1 \mathrm{H}, \mathrm{H}-1), 2.95$ (dd, $J=10.70 \mathrm{~Hz}, J=2.75 \mathrm{~Hz}$, $1 \mathrm{H}, \mathrm{H}-4), 3.00$ (dd, $J=10.05 \mathrm{~Hz}, J=3.49 \mathrm{~Hz}, 1 \mathrm{H}, \mathrm{H}-4), 3.46-3.73\left(\mathrm{dd},{ }^{1} J=94 \mathrm{~Hz},{ }^{2} J=13 \mathrm{~Hz}\right.$, $2 \mathrm{H}, \mathrm{NCH}_{2}$ ), 4.13-4.17 (m, 1H, H-5), 7.16-7.31 (m, 5H, Ar-H); ${ }^{13} \mathrm{C}-\mathrm{NMR}$ (126 MHz, DMSO$\left.\mathrm{d}_{6}\right): \delta=28.1,33.5,42.5,46.1,52.0,54.1,54.6,60.5,81.6,127.4,128.6,138.5,149.6,174.6$; MS: (ESI) $\mathrm{m} / \mathrm{z}=317.76(\mathrm{M}+1)$; Anal. Calcd for $\mathrm{C}_{18} \mathrm{H}_{24} \mathrm{~N}_{2} \mathrm{O}_{3}$ : C, 68.33; H, 7.65; N, 8.85; found: C, 68.30; H, 7.67; N, 8.83. 


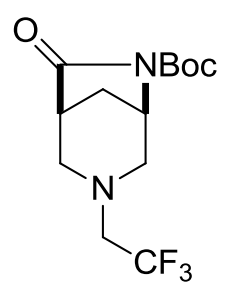

$\left(1 S^{*}, 5 R^{*}\right)-t$-Butyl 7-oxo-3-(2,2,2-trifluoroethyl)-3,6-diazabicyclo[3.2.1]octane-6-carboxylate, $( \pm)-41$

Colorless oil; yield: $16 \%(120 \mathrm{mg}) ; R_{\mathrm{f}}=0.51$ ( $n$-hexane/EtOAc $\left.1: 1\right) ;{ }^{1} \mathrm{H}-\mathrm{NMR}(500 \mathrm{MHz}$, $\left.\mathrm{CDCl}_{3}\right): \delta=1.53\left(\mathrm{~s}, 9 \mathrm{H}, \mathrm{CH}_{3}\right), 1.61-1.65(\mathrm{~m}, 1 \mathrm{H}, \mathrm{H}-8), 2.21-2.28(\mathrm{~m}, 1 \mathrm{H}, \mathrm{H}-8), 2.56-2.61(\mathrm{~m}$, 1H, H-1), 2.82 (d, $J=11.08$ Hz, 1H, H-2), 2.90 (d, $J=10.41 \mathrm{~Hz}, 1 \mathrm{H}, \mathrm{H}-2), 3.04-3.26$ (m, 4H, $\left.\mathrm{H}-4, \mathrm{NCH}_{2}\right), 4.26-4.31$ (m, 1H, H-5); ${ }^{13} \mathrm{C}-\mathrm{NMR}\left(126 \mathrm{MHz}, \mathrm{CDCl}_{3}\right): \delta=28.0,33.1,42.6,52.3$, 53.2, 54.4, $56.9\left(\mathrm{q},{ }^{2} J_{\mathrm{C}, \mathrm{F}}=30.7 \mathrm{~Hz}, \mathrm{CCF}_{3}\right), 82.9,125.8\left({ }^{1} J_{\mathrm{C}, \mathrm{F}}=285.8 \mathrm{~Hz}, \mathrm{CF}_{3}\right), 149.6,174.5$; ${ }^{19} \mathrm{~F}-\mathrm{NMR}\left(471 \mathrm{MHz}, \mathrm{CDCl}_{3}\right): \delta=-68.2(\mathrm{t}, J=9.4 \mathrm{~Hz}$ ); MS: (ESI) $\mathrm{m} / \mathrm{z}=331.74(\mathrm{M}+23)$; Anal. Calcd for $\mathrm{C}_{13} \mathrm{H}_{19} \mathrm{~F}_{3} \mathrm{~N}_{2} \mathrm{O}_{3}$ : C, 50.65; H, 6.21; N, 9.09; found: C, 50.68; H, 6.20; F, 18.48; N, 9.07.

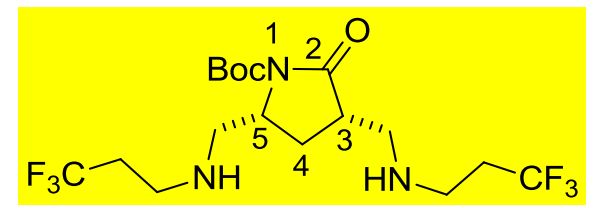

$\left(3 R^{*}, 5 S^{*}\right)-t$-Butyl 2-oxo-3,5-bis(((3,3,3-trifluoropropyl)amino)methyl)pyrrolidine-1-carboxylate, $( \pm)-42$

Colorless oil; yield: $9 \%$ (70 mg); $R_{\mathrm{f}}=0.31$ ( $n$-hexane/EtOAc 1:1); ${ }^{1} \mathrm{H}-\mathrm{NMR}(500 \mathrm{MHz}, \mathrm{DMSO}-$ $\left.\mathrm{d}_{6}\right): \delta=1.43\left(\mathrm{~s}, 9 \mathrm{H}, \mathrm{CH}_{3}\right), 1.57-1.62(\mathrm{~m}, 1 \mathrm{H}, \mathrm{H}-4), 2.07-2.15(\mathrm{~m}, 1 \mathrm{H}, \mathrm{H}-4), 2.24$ (d, $J=10.75$ $\left.\mathrm{Hz}, 1 \mathrm{H}, \mathrm{CH}_{2} \mathrm{CF}_{3}\right), 2.31-2.36\left(\mathrm{~m}, 1 \mathrm{H}, \mathrm{CH}_{2} \mathrm{CF}_{3}\right), 2.35-2.45\left(\mathrm{~m}, 2 \mathrm{H}, \mathrm{CH}_{2} \mathrm{CF}_{3}\right), 2.45-2.49$ (m, $1 \mathrm{H}$, $\mathrm{H}-3), 2.52-2.60\left(\mathrm{~m}, 2 \mathrm{H}, \mathrm{NHCH}_{2}\right), 2.61-2.69$ (m, 2H, $\left.\mathrm{NHCH}_{2}\right), 2.70-2.77$ (m, 2H, $\mathrm{NHCH}_{2}$ ), 2.92-3.08 (m, 2H, $\mathrm{NHCH}_{2}$ ), 4.18-4.23 (m, 1H, H-5), 6.17-6.28 (brs. 2H, NH); ${ }^{13} \mathrm{C}-\mathrm{NMR}(126$ MHz, DMSO-d $)_{6}: \delta=28.1,30.0$ and 30.2 and 30.4 and $30.6\left(\mathrm{q},{ }^{2} J_{\mathrm{C}, \mathrm{F}}=26.5 \mathrm{~Hz}, \mathrm{CCF}_{3}\right.$ ), 31.72 and 31.94 and 32.17 and $32.40\left(\mathrm{q},{ }^{2} J_{\mathrm{C}, \mathrm{F}}=28.5 \mathrm{~Hz}, \mathrm{CCF}_{3}\right), 33.4,39.2$ and $39.3\left(\mathrm{q},{ }^{3} J_{\mathrm{C}, \mathrm{F}}=4.1\right.$ $\mathrm{Hz}, \mathrm{CCH}_{2} \mathrm{CF}_{3}$ ), 42.3, 49.1 and 49.2 (q, ${ }^{3} J_{\mathrm{C}, \mathrm{F}}=3.7 \mathrm{~Hz}, \mathrm{CCH}_{2} \mathrm{CF}_{3}$ ), 52.0, 53.3, 54.5, 81.7, 125.4 and $127.6\left({ }^{1} J_{\mathrm{C}, \mathrm{F}}=279.7 \mathrm{~Hz}, \mathrm{CF}_{3}\right), 126.5\left({ }^{1} J_{\mathrm{C}, \mathrm{F}}=279.7 \mathrm{~Hz}, \mathrm{CF}_{3}\right), 149.5,174.3 ;{ }^{19} \mathrm{~F}-\mathrm{NMR}(471$ MHz, DMSO-d 6$): \delta=-63.4(\mathrm{t}, J=11.8 \mathrm{~Hz}),-64.4(\mathrm{t}, J=11.8 \mathrm{~Hz})$; Anal. Calcd for $\mathrm{C}_{17} \mathrm{H}_{27} \mathrm{~F}_{6} \mathrm{~N}_{3} \mathrm{O}_{3}$ : C, 46.89; H, 6.25; N, 9.65; found: C, 46.92; H, 6.23; F, 26.19; N, 9.67. 
<smiles>CCOC(=O)C1=CN(CC(F)(F)F)[C@@H](OCC)C(NC(=O)c2ccccc2)C1</smiles>

$\left(5 R^{*}, 6 S^{*}\right)$-Ethyl 5-benzamido-6-ethoxy-1-(3,3,3-trifluoropropyl)-1,4,5,6-tetrahydropyridine-3-carboxylate, $( \pm)-21$ a

White solid, mp: $199-200{ }^{\circ} \mathrm{C}$; yield: $30 \%(0.11 \mathrm{~g}) ; R_{\mathrm{f}}=0.30(n$-hexane/EtOAc $1: 2) ;{ }^{1} \mathrm{H}-\mathrm{NMR}$ (500 MHz, DMSO-d 6 ): $\delta=1.10\left(\mathrm{t}, J=7.20 \mathrm{~Hz}, 3 \mathrm{H}, \mathrm{CH}_{3}\right), 1.23\left(\mathrm{t}, J=7.22 \mathrm{~Hz}, 3 \mathrm{H}, \mathrm{CH}_{3}\right), 2.39$ $2.48\left(\mathrm{~m}, 2 \mathrm{H}, \mathrm{CH}_{2}\right), 3.52-3.61\left(\mathrm{~m}, 2 \mathrm{H}, \mathrm{OCH}_{2}\right), 3.95-4.01(\mathrm{~m}, 1 \mathrm{H}, \mathrm{H}-5), 4.11-4.18(\mathrm{~m}, 2 \mathrm{H}$, $\mathrm{OCH}_{2}$ ), 4.20-4.26 (m, 1H, $\mathrm{CH}_{2} \mathrm{CF}_{3}$ ), 4.30-4.37 (m, 1H, $\mathrm{CH}_{2} \mathrm{CF}_{3}$ ), 4.78-4.81 (m, 1H, H-6), 7.28 (s, H-2), 7.45-7.56 (m, 3H, Ar-H), 7.88-8.00 (m, 2H, Ar-H), 8.40 (brs, 1H, N-H); ${ }^{13} \mathrm{C}-\mathrm{NMR}$ $\left(126 \mathrm{MHz}, \mathrm{DMSO}-\mathrm{d}_{6}\right): \delta=15.0,15.8,21.8,48.2,53.6\left(\mathrm{q},{ }^{2} J_{\mathrm{C}, \mathrm{F}}=31.5 \mathrm{~Hz}, \mathrm{CCF}_{3}\right), 59.4,64.8$, 85.4, 99.0, $125.2\left(\mathrm{q},{ }^{1} J_{\mathrm{C}, \mathrm{F}}=281.4 \mathrm{~Hz}, \mathrm{CF}_{3}\right), 127.9,128.7,131.8,134.6,142.7,166.7,166.9 ;{ }^{19} \mathrm{~F}-$ NMR (471 MHz, DMSO-d $)$ ): $\delta=-72.2 \mathrm{~Hz}$; Anal. Calcd for $\mathrm{C}_{20} \mathrm{H}_{25} \mathrm{~F}_{3} \mathrm{~N}_{2} \mathrm{O}_{4}$ : C, 57.96; H, 6.08; N, 6.76; found: C, 57.65; H, 6.32; N, 6.99;<smiles>CCOC(=O)C(CNc1ccccc1)CC(CNc1ccccc1)NC(=O)c1ccccc1</smiles>

$\left(2 S^{*}, 4 R^{*}\right)$-Ethyl 4-benzamido-5-(phenylamino)-2-((phenylamino)methyl)pentanoate, $( \pm)$ 29a

White solid, mp:110-112 ${ }^{\circ} \mathrm{C}$; yield: $29 \%(0.12 \mathrm{~g}) ; R_{\mathrm{f}}=0.33$ (n-hexane/EtOAc 1:2); ${ }^{1} \mathrm{H}-\mathrm{NMR}$ (500 MHz, DMSO-d $\left.)_{6}\right): \delta=1.15\left(\mathrm{t}, J=7.20 \mathrm{~Hz}, 3 \mathrm{H}, \mathrm{CH}_{3}\right), 1.47-1.53\left(\mathrm{~m}, 1 \mathrm{H}, \mathrm{CH}_{2}\right), 1.82-1.98$ (m, $2 \mathrm{H}, \mathrm{CH}_{2}$ and $\left.\mathrm{H}-2\right), 2.04-2.10\left(1 \mathrm{H}, \mathrm{NCH}_{2}\right), 2.76-2.83\left(\mathrm{~m}, 1 \mathrm{H}, \mathrm{NCH}_{2}\right), 2.83-3.02(\mathrm{~m}, 2 \mathrm{H}$, $\mathrm{NCH}_{2}$ ), 3.53-3.57 (m, 2H, $\left.\mathrm{CH}_{2} \mathrm{Ph}\right), 3.68-3.76\left(\mathrm{~m}, 2 \mathrm{H}, \mathrm{CH}_{2} \mathrm{Ph}\right), 3.92-4.07$ (m, 3H, H-4 and $\mathrm{OCH}_{2}$ ), 6.41 (brs, 2H, NH), 7.30-7.53 (m, 13H), 7.78-7.82 (m, 2H, Ar-H), 8.43 (brs, NH); ${ }^{13} \mathrm{C}-$ NMR (126 MHz, DMSO-d 6 ): $\delta=14.5,32.8,41.1,46.1,50.1,54.6,57.7,60.6,52.1,127.7$, 127.8, 128.3 128.6, 128.7, 128.8, 129.0, 129.1, 129.3, 131.6, 134.8, 136.4, 138.3, 166.2, 173.2; Anal. Calcd for $\mathrm{C}_{27} \mathrm{H}_{31} \mathrm{~N}_{3} \mathrm{O}_{3}$ : C, 72.78; H, 7.01; N, 9.43; found: C, 72.50; H, 6.75; N, 9.08.

\section{Acknowledgments}

We are grateful to the Hungarian Research Foundation (NKFIH Nos. K 115731, K 129049 and K 119282) for financial support. The financial support of the GINOP-2.3.2-152016-00014 project is also acknowledged. This research was supported by the EU-funded 
Hungarian grant EFOP-3.6.1-16-2016-00008. Ministry of Human Capacities, Hungary grant 20391-3/2018/FEKUSTRAT is also acknowledged.

\section{References}

1. (a) M. Ordonez, C. Cativiela, R. E. Ivan, Tetrahedron: Asymmetry 2016, 27, 999-1055; (b) X. Wang, M. Ma, A. Gopi K. Reddy, W. Hu, Tetrahedron 2017, 73, 1381-1388; (c) H. Awada, S. Robin, R. Guillot, O. Yazbeck, D. Naoufal, N. Jaber, A. Hachem, D. J. Aitken, Eur. J. Org. Chem. 2014, 7148-7155; (d) K. E. S. Locock, I. Yamamoto, P. Tran, J. R. Hanrahan, M. Chebib, G. A. R. Johnston, R. D. Allan, J. Med. Chem. 2013 56, 5626-5630; (e) Y. Tahara, M. Michino, M. Ito, K. S. Kanyivab, T. Shibata, Chem. Commun. 2015, 51, 16660-16663; (f) M. Ordonez, C. Cativiela, Tetrahedron: Asymmetry 2007, 18, 3-99; (g) A. Pinto, L. Tamborini, E. Pennacchietti, A. Coluccia, R. Silvestri, G. Cullia, C. De Micheli, P. Conti, D. De Biase, J. Enzyme Inhib, Med. Chem. 2015, 1475-6374; (h) Amino Acids, Peptides and Proteins in Organic Chemistry, vol. 1, Origins and Synthesis of Amino Acids. Edited by A. B. Hughes, 2009 WILEY-VCH Verlag GmbH \& Co. KGaA, Weinheim; (i) Amino Acids, Peptides and Proteins in Organic Chemistry, vol. 2, Modified Amino Acids, Organocatalysis and Enzymes. Edited by A. B. Hughes, 2009 WILEY-VCH Verlag GmbH \& Co. KGaA, Weinheim.

2. (a) N. Rodríguez-Vázquez, S. Salzinger, L. F. Silva, M. Amorín, J. R. Granja, Eur. J. Org. Chem. 2013, 3477-3493; (b) J. Aguilera, J. A. Cobos, R. Gutiérrez-Abad, C. Acosta, P. Nolis, O. Illa, R. M. Ortuño, Eur. J. Org. Chem. 2013, 3494-3503; (c) Y. Shi, P. Teng, P. Sang, F. She, L. Wei, J. Cai, Acc. Chem. Res. 2016, 49, 428-441.

3. (a) J. Wang, M. Sánchez-Roselló, J. L: Aceña, C. del Pozo, A. E. Sorochinsky, S. Fustero, V. A: Soloshonok, H. Liu, Chem. Rev. 2014, 114, 2432. (b) Fluorine in Pharmaceutical and Medicinal Chemistry: From Biophysical Aspects to Clinical Applications, Imperial College Press, London 2012. Edited by Gouverneur, V. and Müller, K.; (c) Y. Zhou, Y. Wang, Z. Gu, S. Wang, W. Zhu, J. L. Acena, V. A: Soloshonok, K. Izawa, H. Liu, Chem. Rev. 2016, $116,442$.

4. (a) R. B. Silverman, J. Med. Chem. 2012, 55, 567-575; (b) H. Lu, R. B. Silverman, J. Med. Chem. 2006, 49, 7404-7412; (c) J. I. Juncosa, A. P. Groves, G. Xia, R. B. Silverman, Bioorg. Med. Chem. 2013, 21, 903-911.

5. (a) E. Erba, C. La Rosa, Tetrahedron 2016, 72, 7975; (b) K. K. C. Liu, S. M. Sakya, C. J. O’Donnell, A. C. Flick, H. X. Ding, Bioorg. Med. Chem. 2012, 20, 1155; (c) D. C. Chiu, T. C. Lin, W. I. Huang, T. J. Cheng, K. C. Tsaic, J. M. Fang, Org. Biomol. Chem. 2017, 15, 
9910-9922; (d) P. C. Wang, D. Chi Chiu, J. T. Jan, W. Huang, Y. C. Tseng, T. T. Li, T. J. Cheng, K. C. Tsai, J. M. Fang, Eur. J. Med. Chem. 2018, 145, 224-234; (e) L. Kiss, M. Nonn, F. Fülöp, Synthesis 2012, 44, 1951-1963.

6. (a) R. Singh, R. Vince, Chem. Rev. 2012, 112, 4642-4686; (b) E. Forró, F. Fülöp, Eur. J. Org. Chem. 2008, 5263-5268; (c) S. Zhu, L. Ren, S. Yu, C. Gong, D. Song, G. Zheng, Bioorg. Med. Chem. Lett. 2014, 24, 4899-4902; (d) S. Karlsson,, P. Cornwall, A. Cruz, F. Pontén, M. Fridén-Saxin, A. Turner Org. Process Res. Dev. 2018, 22, 337-343.

7. (a) Z. Galla, E. Forró, F. Fülöp, Tetrahedron: Asymmetry 2016, 27, 729-731; (b) G. A. Boyle, C. D. Edlin, Y. Li, D. C. Liotta, G. L. Morgans, C. C. Musonda, Org. Biomol. Chem. 2012, 10, 1870-1876; (c) U. S. Singh, R. C. Mishra, R. Shankar, C. K. Chu, J. Org. Chem. 2014, 79, 3917-3923.

8. (a) Amino Acids, Peptides and Proteins in Organic Chemistry, vol.3, Building Blocks, Catalysis and Coupling Chemistry. Edited by A. B. Hughes, 2011 WILEY-VCH Verlag GmbH \& Co. KGaA, Weinheim; (b) Amino Acids, Peptides and Proteins in Organic Chemistry. Vol.4, Protection Reactions, Medicinal Chemistry, Combinatorial Synthesis. Edited by A. B. Hughes, 2011 WILEY-VCH Verlag GmbH \& Co. KGaA, Weinheim; (c) M. A. Blaskovich, Handbook on syntheses of amino acids: General routes for the syntheses of amino acids; Oxford University Press: Oxford; New York, 2010; (d) C. Cativiela, M. Ordóñez, Tetrahedron: Asymmetry 2009, 20,1-63; (e) M. A. T. Blaskovich J. Med. Chem. 2016, 59, 10807-10836.

9. (a) L. Kiss, F. Fülöp, Chem. Rev. 2014, 114, 1116-1169; (b) L. Kiss, I. M. Mándity, F. Fülöp, Amino Acids 2017, 49, 1441-1455; (c) R. A. Ábrahámi, L. Kiss, S. Fustero, F. Fülöp, Synthesis 2017, 49, 1206-1213.

10. (a) R. A. Ábrahámi, S. Fustero, F. Fülöp, L. Kiss, Synlett 2018, 29, 2066-2070; R. A. Ábrahámi, L. Kiss, P. Barrio, F. Fülöp, Tetrahedron 2016, 72, 7526-7535.

11. L. Kiss, E. Forró, R. Sillanpää, F. Fülöp, Synthesis, 2010, 1, 153-160.

12. U. S. Singh, R. C. Mishra, R. Shankar, C. K. Chu, J. Org. Chem. 2014, 79, 3917-3923. 